\title{
Pre-Test Assessment of the Upper Bound of the Drag Coefficient Repeatability of a Wind Tunnel Model
}

\author{
N. Ulbrich ${ }^{\dagger}$ and A. L'Esperance \\ Jacobs Technology Inc., Moffett Field, California 94035
}

\begin{abstract}
A new method is presented that computes a pre-test estimate of the upper bound of the drag coefficient repeatability of a wind tunnel model. This upper bound is a conservative estimate of the precision error of the drag coefficient. For clarity, precision error contributions associated with the measurement of the dynamic pressure are analyzed separately from those that are associated with the measurement of the aerodynamic loads. The upper bound is computed by using information about the model, the tunnel conditions, and the balance in combination with an estimate of the expected output variations as input. The model information consists of the reference area and an assumed angle of attack. The tunnel conditions are described by the Mach number and the total pressure or unit Reynolds number. The balance inputs are the partial derivatives of the axial and normal force with respect to all balance outputs. Finally, an empirical output variation of $1.0 \mathrm{microV} / \mathrm{V}$ is used to relate both random instrumentation and angle measurement errors to the precision error of the drag coefficient. Results of the analysis are reported by plotting the upper bound of the precision error versus the tunnel conditions. The analysis shows that the influence of the dynamic pressure measurement error on the precision error of the drag coefficient is often small when compared with the influence of errors that are associated with the load measurements. Consequently, the sensitivities of the axial and normal force gages of the balance have a significant influence on the overall magnitude of the drag coefficient's precision error. Therefore, results of the error analysis can be used for balance selection purposes as the drag prediction characteristics of balances of similar size and capacities can objectively be compared. Data from two wind tunnel models and three balances are used to illustrate the assessment of the precision error of the drag coefficient.
\end{abstract}

\section{Nomenclature}

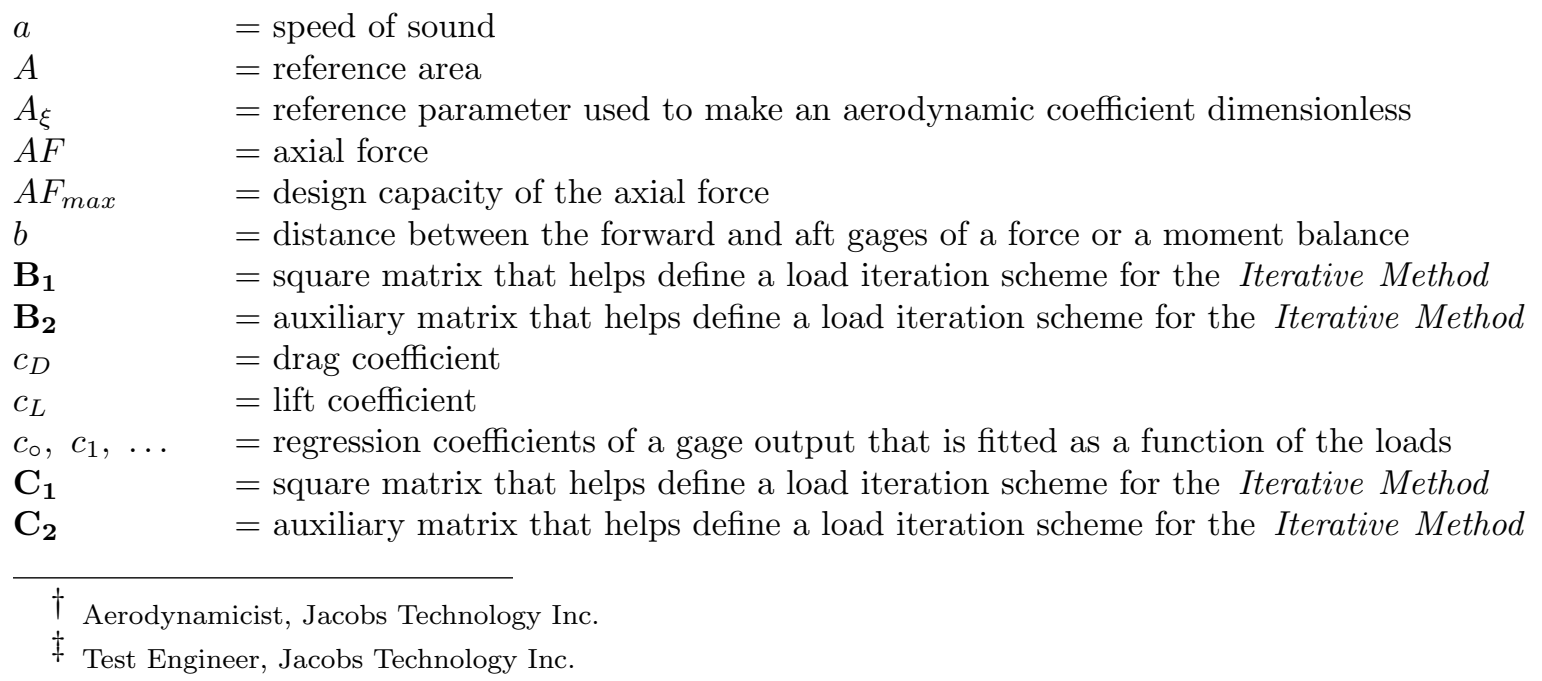




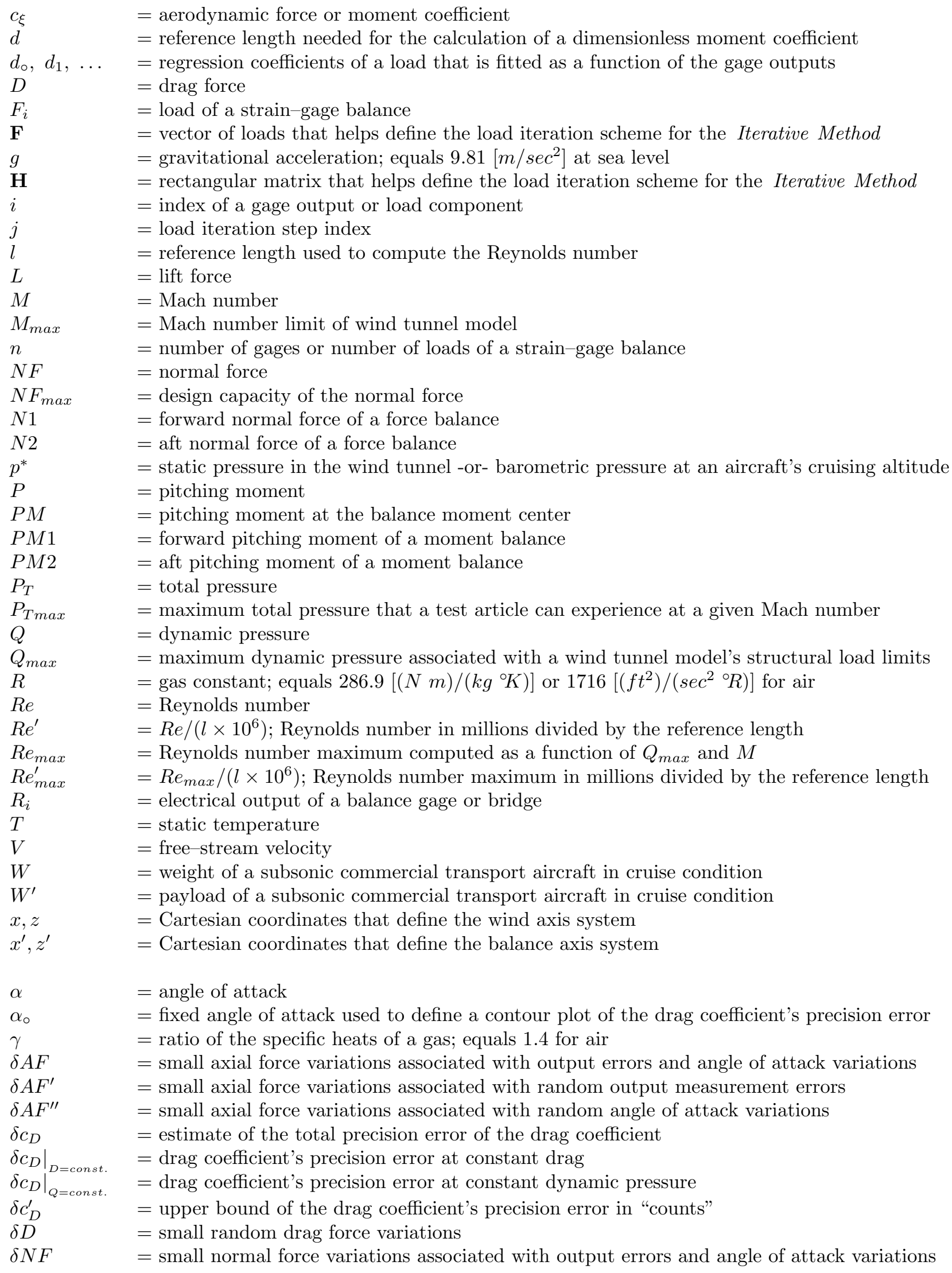




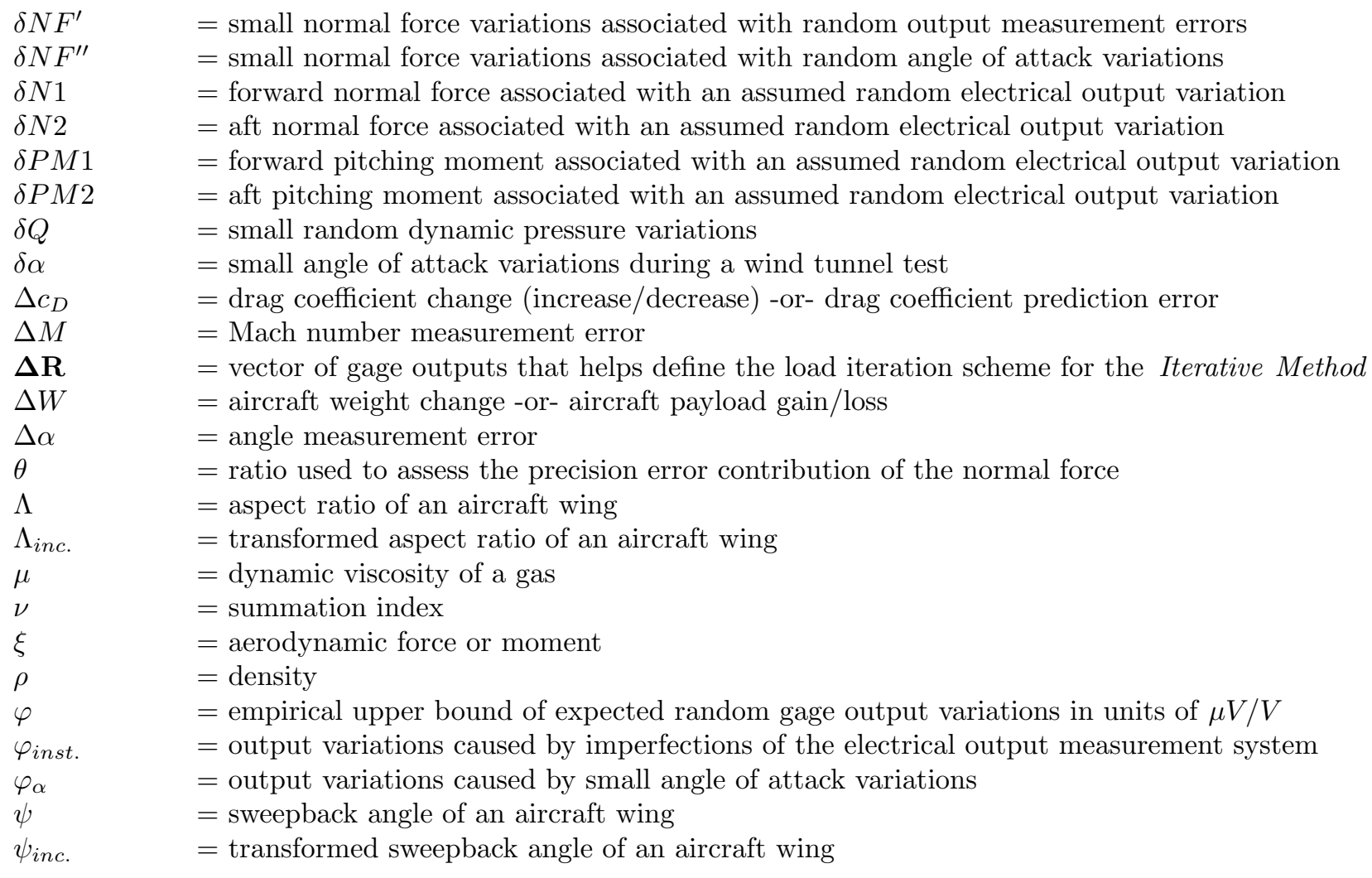

\section{Introduction}

The precise determination of the drag coefficient plays an important role during performance tests of wind tunnel models at high subsonic Mach numbers as the impact of small model configuration changes on the drag coefficient needs to be identified with great confidence. In addition, the "repeatability" of the drag coefficient, i.e., the difference between drag coefficients of repeat data points, needs to be as small as possible so that observed performance improvements of a specific test article configuration can "objectively" be reproduced months or years after they were first implemented. It can be shown, for example, that a one "count" (0.0001) reduction of the drag coefficient of a subsonic commercial transport aircraft operating in cruise condition can directly result in a $1 \%$ payload increase (see App. 1 for more details). This significant improvement may not be detectable if the error of the wind tunnel's Mach number measurement system exceeds 0.001 (see App. 2). Therefore, the Mach number measurement system of a production wind tunnel is often designed such that the Mach number uncertainty is well below 0.001 for most of its operating envelope (Fig. 1 below shows Mach number uncertainty estimates for the NASA Ames 11-ft Transonic Wind Tunnel as an example). Similarly, the angle of attack measurement error should be less than 0.02 degrees so that drag coefficient changes on the order of one "count" can be observed with confidence (concluded from App. 3). Some wind tunnel operators also like to closely monitor the "repeatability" of the drag coefficient during wind tunnel tests because it is an indirect indicator of the overall quality of the recorded test data.

In general, the "repeatability" of the drag coefficient can be quantified by the precision (random) error that is contained in the drag coefficient prediction itself. Several factors influence the magnitude of the observed precision error of the drag coefficient during a wind tunnel test (e.g., balance design and performance, support system motion characteristics, steadiness of the dynamic pressure and angle measurements, data sampling rates, etc.). Consequently, an effective control of the drag coefficient's precision error has to minimize random changes of the most important measurements that contribute to unwanted variations of the computed drag coefficient. In addition, pre-test estimates of the drag coefficient's precision error would be very useful for test planning purposes as they would give wind tunnel customers a better idea (i) which part of a tunnel's operating envelope and (ii) what specific combination of strain-gage balance characteristics will best support their test objectives. Therefore, the authors developed a new method that uses some readily 
available information about the wind tunnel model, the balance, and the wind tunnel's operating envelope for a realistic pre-test assessment of the drag coefficient's precision error.
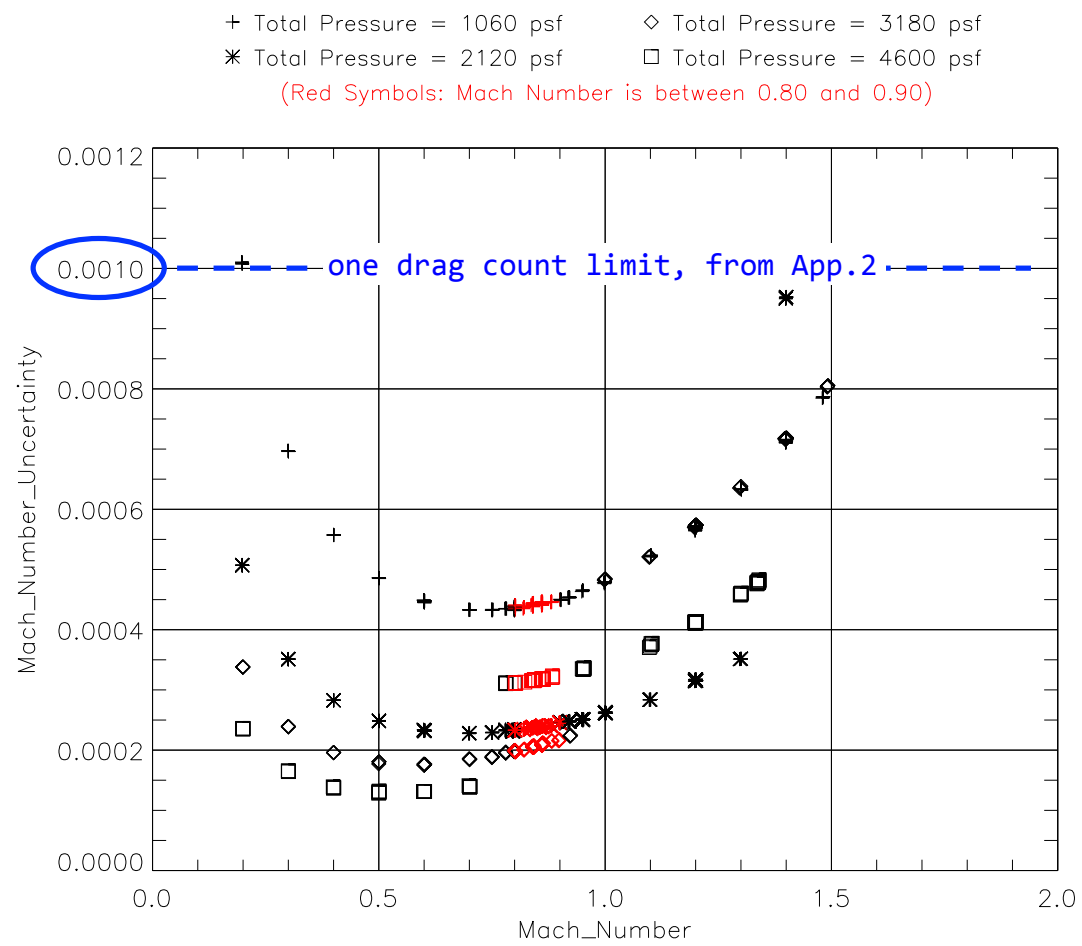

Fig. 1 Mach number uncertainty of the NASA Ames 11-ft Transonic Wind Tunnel (taken from Ref. [3]).

Details of the proposed assessment of the drag coefficient's precision error are discussed in the next section of the paper. Afterwards, results for two wind tunnel test configurations are used to illustrate the assessment of the drag coefficient's precision error for models that are tested in a pressurized wind tunnel.

\section{Precision Error Assessment}

\section{A. Upper Bound of the Precision Error}

The derivation of a pre-test estimate of the precision error of the drag coefficient of a wind tunnel model is discussed in detail in this section. The goal of the precision error analysis is the identification of a realistic upper bound of the precision error of the drag coefficient that is based on "objective" information about test article, balance, and tunnel conditions. In other words, only a minimum amount of empirical information should be used as input for the identification of the upper bound of the drag coefficient's precision error.

The derivation of an estimate of the upper bound of the drag coefficient's precision error starts with the general definition of the drag coefficient. This dimensionless quantity is defined as follows

$$
c_{D}(D, Q)=\frac{D}{Q \cdot A}
$$

where $D$ is the measured aerodynamic drag force on the model, $Q$ is the dynamic pressure, and $A$ is the model's reference area (Ref. [1]). The drag coefficient is a function of two independent variables, i.e., of the drag force $D$ and the dynamic pressure $Q$, as the model's reference area $A$ is a constant for a given test article. Consequently, small changes of the drag coefficient can mathematically be described by its total differential (Ref. [2]). It is given by the expression

$$
d c_{D}=\frac{\partial c_{D}}{\partial D} \cdot d D+\frac{\partial c_{D}}{\partial Q} \cdot d Q
$$

where $d c_{D}, d D$, and $d Q$ represent small changes of related variables. Now, assuming that small "random" changes of (i) the tunnel conditions, (ii) the model position/orientation in the test section, and (iii) the 
electrical outputs of the balance gages are primarily responsible for the fact that the drag coefficients of two repeat points differ by a small amount, it is possible to relate them to small changes of the two independent variables. Then, an upper bound of the drag coefficient's precision error $\delta c_{D}$ can be defined by using the absolute values of the partial derivatives of the drag coefficient in combination with the absolute value of small changes of both the measured drag force $(\delta D)$ and the dynamic pressure $(\delta Q)$. Consequently, we get the following conservative estimate of the upper bound of the drag coefficient's precision error:

$$
\delta c_{D} \leq\left|\frac{\partial c_{D}}{\partial D}\right| \cdot|\delta D|+\left|\frac{\partial c_{D}}{\partial Q}\right| \cdot|\delta Q|
$$

Absolute values of the partial derivatives and the small changes of the independent variables have to be used in Eq. (3) because the signs of $\delta D$ and $\delta Q$ are assumed to be unknown. The two partial derivatives can easily be computed. We get:

$$
\begin{aligned}
&\left|\frac{\partial c_{D}}{\partial D}\right|=\frac{1}{Q \cdot A} \\
&\left|\frac{\partial c_{D}}{\partial Q}\right|=\left|\frac{D}{A} \cdot \frac{(-1)}{Q^{2}}\right|=\left|\frac{D}{Q \cdot A}\right| \cdot \frac{1}{Q}=\left|c_{D}\right| \cdot \frac{1}{Q}
\end{aligned}
$$

Then, after using the right-hand sides of Eqs. $(4 a)$ and $(4 b)$ to replace the partial derivatives used in Eq. (3), we get the following upper bound of the drag coefficient's precision error:

\section{UPPER BOUND OF PRECISION ERROR OF DRAG COEFFICIENT}

$$
\begin{aligned}
\delta c_{D} \leq\left.\delta c_{D}\right|_{Q=\text { const. }}+\left.\delta c_{D}\right|_{D=\text { const. }} \\
\text { where } \\
\text { balance and/or support system choice }\left.\Longrightarrow \delta c_{D}\right|_{Q=\text { const. }} \leq \frac{1}{Q \cdot A} \cdot|\delta D| \\
\text { tunnel condition choice }\left.\Longrightarrow \delta c_{D}\right|_{D=\text { const. }} \leq\left|c_{D}\right| \cdot\left|\frac{\delta Q}{Q}\right|
\end{aligned}
$$

The above estimate of the upper bound of the drag coefficient's precision error clearly identifies the two major parameter that control the overall magnitude of the drag coefficient's precision error:

$$
\begin{aligned}
|\delta D| & \Longrightarrow \text { variations of the drag force measurement at constant dynamic pressure } \\
|\delta Q / Q| & \Longrightarrow \text { relative error of the dynamic pressure measurement of the tunnel }
\end{aligned}
$$

The first parameter $(|\delta D|)$ depends on (i) the balance characteristics and (ii) the angle of attack measurement error on the model. Therefore, for example, the selection of (i) an alternate balance or (ii) a more rigid model support system can be used to influence the drag coefficient's precision error. The second parameter $(|\delta Q / Q|)$ depends on the dynamic pressure measurement error at the model location in the test section, i.e., on the error that is associated with the measurement of the tunnel conditions. It can also be used to influence the drag coefficient's precision error as the relative error of the dynamic pressure measurement often decreases with increasing dynamic pressure. - In the next sections of the paper the drag coefficient's precision error at constant drag and at constant dynamic pressure are investigated in more detail.

\section{B. Precision Error at Constant Drag}

The drag coefficient's precision error at constant drag is related to the tunnel condition choice as the relative error of the dynamic pressure measurement is directly proportional to the drag coefficient's precision error at constant drag (see Eq. $(5 c)$ ). Unfortunately, the determination of the relative error $\delta Q / Q$ of the 
dynamic pressure of a wind tunnel facility can be complex because it highly depends on the facility's pressure \& temperature instrumentation hardware setup and the operating envelope of the tunnel. Therefore, the authors decided to use existing uncertainty analysis results for the NASA Ames 11-ft Transonic Wind Tunnel (TWT) in order to get a realistic estimate of the precision error at constant drag.

A rigorous uncertainty analysis of the dynamic pressure measurement of the NASA Ames 11-ft TWT was performed in 2003/2004. Results of this analysis were published in Ref. [3]. Figure 2 below shows an alternate representation of the uncertainty analysis results for the dynamic pressure that are given in Ref. [3].
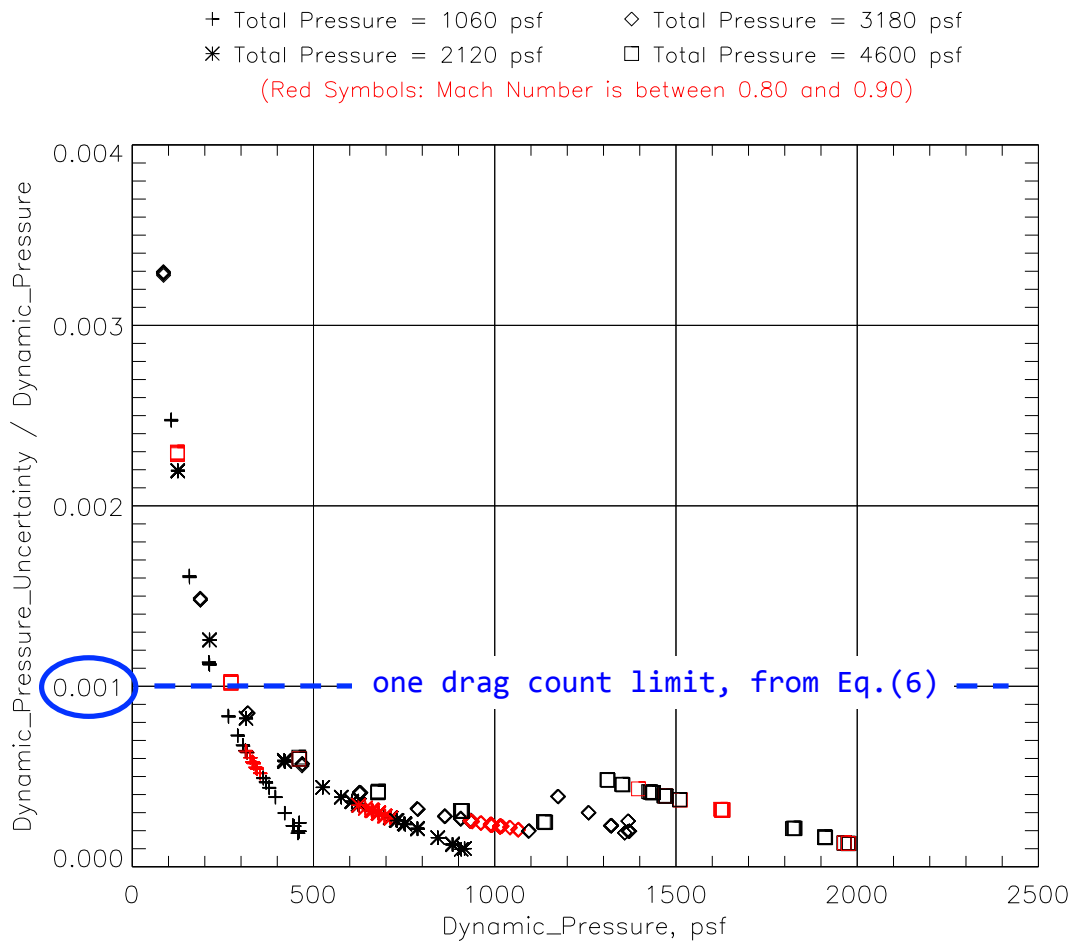

Fig. 2 Relative error estimate of the dynamic pressure of the NASA Ames 11-ft Transonic Wind Tunnel.

In this case, the dimensionless dynamic pressure uncertainty is plotted versus the dynamic pressure itself as the dimensionless uncertainty value can be interpreted as a conservative estimate of the relative error of the dynamic pressure measurement. It can be concluded from Fig. 2 that the relative error of the tunnel's dynamic pressure measurement is well below 0.001 for most of the tunnel's operating envelope. In addition, it is known that the drag coefficient maximum for most wind tunnel models is near 0.1. Therefore, the following estimate of the upper bound of the drag coefficient's precision error at constant drag can be made for the NASA Ames 11-ft TWT:

\section{UPPER BOUND OF PRECISION ERROR AT CONSTANT DRAG}

$$
\left.\delta c_{D}\right|_{D=\text { const. }} \leq\left|c_{D}\right| \cdot|\delta Q / Q| \leq 0.1 \times 0.001=0.0001 \equiv \text { one drag count }
$$

The drag coefficient of a typical subsonic commercial transport aircraft operating in cruise condition is $c_{D} \approx 0.04$ (equals a conservative upper limit of estimates that are given in App. 1, Table 6). In addition, it is concluded from Fig. 2, that the relative error $|\delta Q / Q|$ of the dynamic pressure measurement for the Ames 11-ft TWT is between 0.0003 and 0.0005 for Mach numbers between 0.8 and 0.9 (see red symbols in Fig. 2). Consequently, the contribution of the dynamic pressure measurement uncertainty to the drag coefficient's precision error is negligible for this assumed cruise condition as it is estimated to be between $1 / 10$ and $1 / 5$ drag count $(0.04 \times 0.0003=0.000012$ or $1 / 10$ count $; 0.04 \times 0.0005=0.000020$ or $1 / 5$ count $)$. In the next 
section, the drag coefficient's precision error at constant dynamic pressure is discussed in more detail.

\section{Precision Error at Constant Dynamic Pressure}

The authors decided to focus their investigations on the precision error contribution $|\delta D|$ that is associated with the drag force measurement itself. This contribution primarily depends on load resolution characteristics, i.e., on the normal and axial force sensitivities, of the chosen balance. It can be used to influence the drag coefficient's precision error as random variations in the drag force measurement can often be reduced by simply selecting a balance with normal and axial force sensitivities for the test that better matches a test article's expected axial and normal force range.

Origins of random variations of the drag force measurement need to be investigated in detail so that the upper bound of the drag coefficient's precision error at constant dynamic pressure can be quantified exactly as defined in Eq. $(5 b)$. Therefore, the relationship between the drag force on the test article and the axial and normal force on the balance needs to be examined. Figure $3 a$ below shows the fundamental connection between the drag force $D$ acting on the test article in the wind axis system (coordinates $x$ and $z$ ) and the balance loads $A F$ and $N F$ that are measured in the balance axis system (coordinates $x^{\prime}$ and $z^{\prime}$ ).

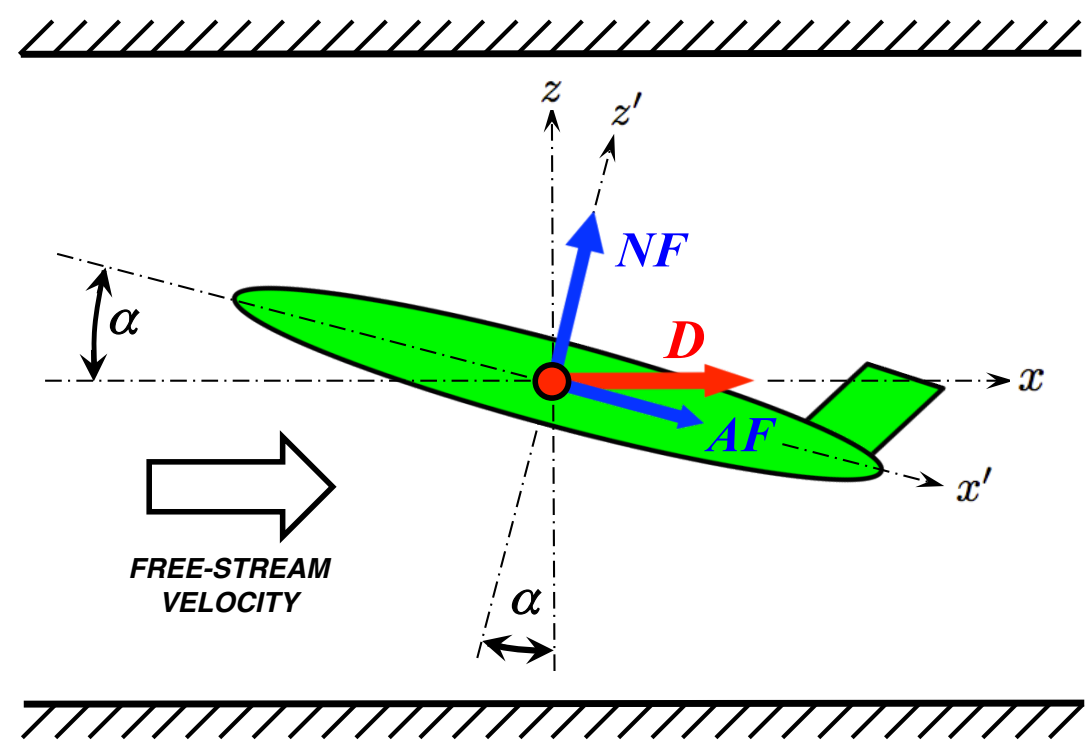

Fig. 3 a Connection between the drag force and the axial and normal force measurements of a balance.

The drag force measurement is a function of three independent variables: the axial force $A F$, the normal force $N F$, and the angle of attack $\alpha$ of the model. It is described by the following relationship:

$$
D=D(A F, N F, \alpha)=A F \cdot \cos \alpha+N F \cdot \sin \alpha
$$

The total differential of the drag force is needed so that an estimate of an upper bound of the variation of the drag force can be obtained. The total differential is given by the following expression:

$$
d D=\frac{\partial D}{\partial A F} \cdot d A F+\frac{\partial D}{\partial N F} \cdot d N F+\frac{\partial D}{\partial \alpha} \cdot d \alpha
$$

The partial derivatives of the drag force can easily be obtained from Eq. (7). We get:

$$
\begin{gathered}
\frac{\partial D}{\partial A F}=\cos \alpha \\
\frac{\partial D}{\partial N F}=\sin \alpha \\
\frac{\partial D}{\partial \alpha}=A F \cdot(-\sin \alpha)+N F \cdot \cos \alpha
\end{gathered}
$$


Then, after substituting the partial derivatives in Eq. (8a) by the right-hand sides of the above three equations and after rearranging the result for clarity, we get:

$$
d D=\{d A F+d \alpha \cdot N F\} \cdot \cos \alpha+\{d N F-d \alpha \cdot A F\} \cdot \sin \alpha
$$

Now, in order to get an inequality for the upper bound of the drag force variation $|\delta D|$, four replacements are made: (i) the differential $d D$ is replaced by the upper bound $|\delta D|$ of the precision error of the drag force measurement, (ii) the differential $d A F$ is replaced by the upper bound $\left|\delta A F^{\prime}\right|$ of the precision error estimate of the axial force measurement at constant angle of attack, (iii) the differential $d N F$ is replaced by the upper bound $\left|\delta N F^{\prime}\right|$ of the precision error estimate of the normal force measurement at constant angle of attack, and (iv) the differential $d \alpha$ is replaced by the upper bound $|\delta \alpha|$ of the precision error of the angle measurement. Consequently, after switching the minus to a plus sign in Eq. (8e) so that a "conservative" estimate is obtained, we get the following upper bound for the absolute value of the drag force variation:

$$
|\delta D| \leq\left\{\left|\delta A F^{\prime}\right|+\left|\delta A F^{\prime \prime}\right|\right\} \cdot \cos |\alpha|+\left\{\left|\delta N F^{\prime}\right|+\left|\delta N F^{\prime \prime}\right|\right\} \cdot \sin |\alpha|
$$

where

$$
\begin{aligned}
\left|\delta A F^{\prime \prime}\right| & =|\delta \alpha| \cdot|N F| \\
\left|\delta N F^{\prime \prime}\right| & =|\delta \alpha| \cdot|A F|
\end{aligned}
$$

It is useful to interpret the right-hand side of Eq. (9a). First, it has to be pointed out that $\left|\delta A F^{\prime}\right|$ and $\left|\delta N F^{\prime}\right|$ are "random" force variations at constant angle of attack and at constant dynamic pressure. Therefore, they are not associated with true "physical" loads that the balance experiences. Instead, they can only be caused by small output measurement errors that result from imperfections of the entire electrical measurement system (gages, wires, amplifiers, etc.). This conclusion can be summarized as follows:

\section{$\left|\delta A F^{\prime}\right| \quad \Longrightarrow \quad$ axial force variation caused by small random output measurement errors \\ $\left|\delta N F^{\prime}\right| \quad \Longrightarrow \quad$ normal force variation caused by small random output measurement errors}

In addition, after reviewing the force diagram shown in Fig. $3 b$ below, it is concluded that the term $\left|\delta A F^{\prime \prime}\right|$ can be interpreted as a small axial force variation at constant normal force.

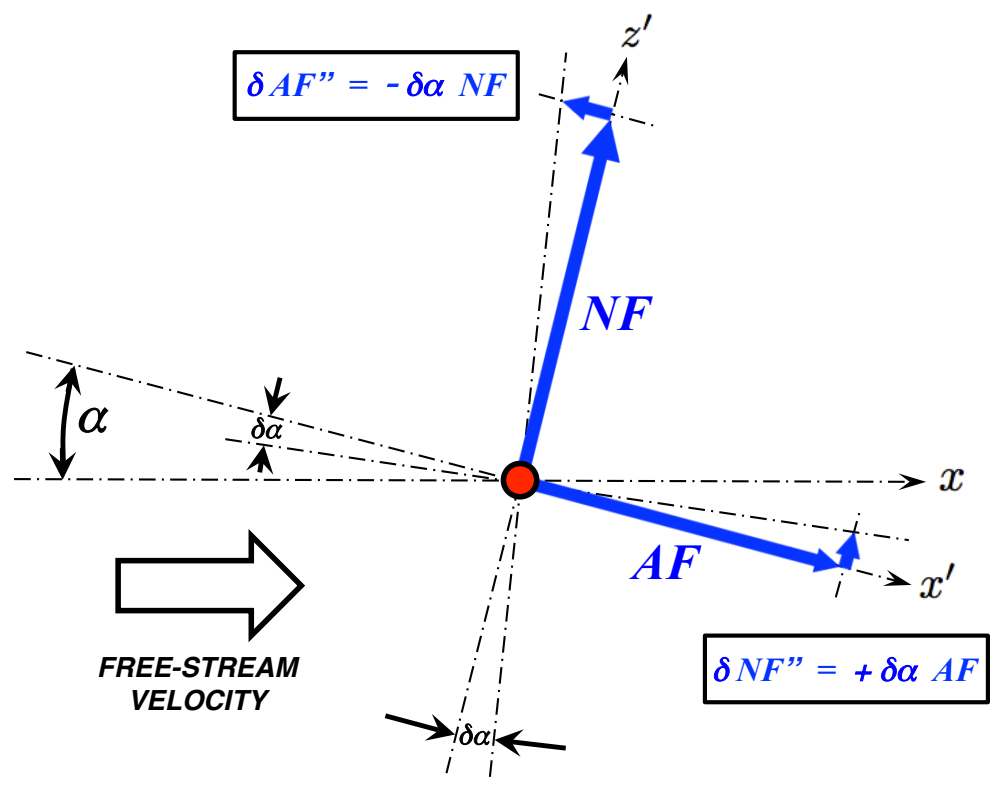

Fig. 36 Connection between angle of attack variations and balance load variations.

Similarly, it is concluded that the term $\left|\delta N F^{\prime \prime}\right|$ can be interpreted as a small normal force variation at constant axial force. Both force variations are associated with true "physical" loads that act on the balance. 
These loads are connected to small "physical" angle of attack changes that occur during "wind-on" runs in a wind tunnel. These angle of attack changes are primarily caused by (i) small "elastic" model support system deformations, (ii) vibrational characteristics of the support system and model/balance assembly, and (iii) imperfections of the facility control system algorithm that sets the requested angle of attack.

The electrical outputs of the balance gages always respond to the sum of $\left|\delta A F^{\prime}\right|$ and $\left|\delta A F^{\prime \prime}\right|$ because "random" output measurement errors and output variations associated with "random" angle of attack variations exist simultaneously. Similarly, the electrical outputs of the balance gages also always respond to the sum of $\left|\delta N F^{\prime}\right|$ and $\left|\delta N F^{\prime \prime}\right|$. Therefore, it makes sense for the current study to combine associated force contributions in single terms. The following definitions can be made:

$$
\begin{aligned}
|\delta A F| & =\left|\delta A F^{\prime}\right|+\left|\delta A F^{\prime \prime}\right| \\
|\delta N F| & =\left|\delta N F^{\prime}\right|+\left|\delta N F^{\prime \prime}\right|
\end{aligned}
$$

Then, inequality $(9 a)$ can be summarized as follows:

$$
|\delta D| \leq|\delta A F| \cdot \cos |\alpha|+|\delta N F| \cdot \sin |\alpha|
$$

Finally, after combining the above inequality with Eq. (5b), we get the following estimate of the upper bound of the drag coefficient's precision error at constant dynamic pressure:

\section{UPPER BOUND OF PRECISION ERROR AT CONSTANT DYNAMIC PRESSURE}

$$
\left.\delta c_{D}\right|_{Q=\text { const. }} \leq \frac{|\delta A F| \cdot \cos |\alpha|+|\delta N F| \cdot \sin |\alpha|}{Q \cdot A}
$$

The numerical evaluation of the right-hand side of Eq. (12) still requires some reasonable estimate of the precision error of the axial and normal force measurements of the strain-gage balance. An estimate of this error is derived in the next section of the paper.

\section{Precision Error of the Balance Loads}

In principle, a reasonable estimate of the precision error of the axial and normal force measurements of the balance is needed so that the right-hand side of Eq. (12) can numerically be evaluated. This estimate can be obtained by combining information about the load resolution properties of the selected strain-gage balance with an empirical estimate of the random variations of the electrical outputs of the gages.

Regression models of balance calibration data are typically used in matrix format in a wind tunnel to predict both the axial and normal force on the balance from the measured gage outputs. This matrix is often called the "Data Reduction Matrix" (see, e.g., Ref. [4], p. 19, p. 62). Some matrix coefficients can be interpreted as least squares approximations of the partial derivatives of a balance load component $F_{i}$ with respect to a gage output $R_{j}$ where $F_{i}$ equals $A F, N F, N 1, N 2, P M 1$, or $P M 2$ depending on the design load format of the strain-gage balance. Therefore, it is possible to define an upper bound of the "random" variations of the axial and normal force by simply multiplying an empirical upper bound of the "random" variation of the electrical outputs with the appropriate set of partial derivatives of the balance load components $F_{i}$ that are needed for the calculation of the axial and normal force of the balance. This "random" variation $\left|\delta F_{i}\right|$ of a balance load component can be described as follows:

$$
\left|\delta F_{i}\right| \approx\left|\frac{\partial F_{i}}{\partial R_{1}}\right| \cdot\left|\delta R_{1}\right|+\ldots+\left|\frac{\partial F_{i}}{\partial R_{n}}\right| \cdot\left|\delta R_{n}\right| \leq|\varphi| \cdot \sum_{\nu=1}^{n} \frac{\partial F_{i}}{\partial R_{\nu}}
$$

In theory, the observed upper bound $|\varphi|$ of the gage output variations consists of two superimposed contributions (i.e., $|\varphi|=\left|\varphi_{\text {inst. }}\right|+\left|\varphi_{\alpha}\right|$ ). The first contribution $\left|\varphi_{\text {inst. }}\right|$ is the result of imperfections of the electrical output measurement system itself. It is associated with the root cause of $\delta A F^{\prime}$ and $\delta N F^{\prime}$. This 
contribution results in "virtual" balance load predictions from the regression model of the calibration data that are not associated with "physical" load changes on the model. The second contribution $\left|\varphi_{\alpha}\right|$ is the result of small angle of attack changes $\delta \alpha$ at constant dynamic pressure. It is related to the root cause of $\delta A F^{\prime \prime}$ and $\delta N F^{\prime \prime}$. This contribution results in load predictions from the regression model of the balance calibration data that are associated with true "physical" load changes on the model. Fortunately, it is known from observed bare balance outputs of many different balance types that $\left|\varphi_{\text {inst. }}\right|$ is much smaller than $\left|\varphi_{\alpha}\right|$. In other words, $\left|\varphi_{\text {inst. }}\right|$ can be neglected for most wind tunnel test situations. Therefore, the authors recommend the following value as the empirical upper bound of the "random" variations of the outputs:

\section{UPPER BOUND OF RANDOM OUTPUT VARIATIONS}

$$
|\varphi| \approx\left|\varphi_{\alpha}\right| \leq 1.0[\mu V / V] \equiv 0.01[\mathrm{deg}] \times(1000[\mu V / V] / 10[\mathrm{deg}])
$$

The choice of $1.0[\mu \mathrm{V} / \mathrm{V}]$ was experimentally confirmed after reviewing repeat data from (i) wind tunnel tests at the NASA Ames 11-ft TWT and (ii) strain-gage balance calibrations. The right-hand side of Eq. (13b) illustrates how the choice of $1.0[\mu \mathrm{V} / \mathrm{V}]$ can be interpreted in a practical context if the relationship between output change and angle of attack change is assumed to be linear. The choice of $1.0[\mu V / V]$ essentially corresponds to an angle measurement variation of $0.01[\mathrm{deg}]$ if the normal/axial force gages have a maximum output of $1000[\mu \mathrm{V} / \mathrm{V}]$ that occurs at an angle of attack of $10[\mathrm{deg}]$.

The recommended upper bound of $1.0[\mu V / V]$ should be multiplied by a reasonable upper bound of the partial derivatives $\partial F_{i} / \partial R_{1}, \ldots, \partial F_{i} / \partial R_{n}$ of the chosen load component $F_{i}$ that is used to compute either the axial or normal force on the wind tunnel model. It is possible, for example, to simply use the sum of the absolute values of the partial derivatives as an upper bound. This choice, however, would be very "conservative" as it implicitly assumes that the sign of all partial derivatives is the same. Instead, a slightly smaller but more realistic upper bound may be obtained by using the definition of the length of a vector in $n$-dimensional space as the upper bound of the partial derivatives. Then, we get:

\section{UPPER BOUND OF THE PARTIAL DERIVATIVES OF A BALANCE LOAD}

$$
S\left(F_{i}, R_{1}, \ldots, R_{n}\right) \leq \sqrt{\left[\frac{\partial F_{i}}{\partial R_{1}}\right]^{2}+\cdots+\left[\frac{\partial F_{i}}{\partial R_{n}}\right]^{2}}=\sqrt{\sum_{\nu=1}^{n}\left[\frac{\partial F_{i}}{\partial R_{\nu}}\right]^{2}}
$$

A typical strain-gage balance uses a single bridge to measure the axial force. Consequently, after combining Eqs. (13b) and (13c) above, the upper bound of the estimated "random" variations of the axial force measurement can be expressed as follow:

$$
|\delta A F| \approx|\varphi| \cdot S\left(A F, R_{1}, \ldots, R_{n}\right)
$$

Similarly, assuming that the balance load prediction matrix describes load components in "direct-read" format, the upper bound of the variations of the normal force measurement can be expressed as follows:

$$
|\delta N F| \approx|\varphi| \cdot S\left(N F, R_{1}, \ldots, R_{n}\right)
$$

Then, after replacing $|\delta A F|$ and $|\delta N F|$ in Eq. (12) by the right-hand sides of Eqs. (14a) and (14b), we get an alternate description of the upper bound of the precision error at constant dynamic pressure:

\section{UPPER BOUND OF PRECISION ERROR AT CONSTANT DYNAMIC PRESSURE}

$$
\left.\delta c_{D}\right|_{Q=\text { const. }} \leq|\varphi| \cdot \frac{S\left(A F, R_{1}, \ldots, R_{n}\right) \cdot \cos |\alpha|+S\left(N F, R_{1}, \ldots, R_{n}\right) \cdot \sin |\alpha|}{Q \cdot A}
$$


A balance load prediction matrix may also be supplied using load components that are described in "force balance" format (see, e.g., Ref. [4] and Ref. [5] for a description of the different balance load formats that are used in the aerospace testing community). Then, the "random" variation of the normal force is estimated by using the following inequality

$$
|\delta N F| \leq|\delta N 1|+|\delta N 2| \approx|\varphi| \cdot\left\{S\left(N 1, R_{1}, \ldots, R_{n}\right)+S\left(N 2, R_{1}, \ldots, R_{n}\right)\right\}
$$

where $N 1$ is the forward normal force component and $N 2$ is the aft normal force component of the balance. Alternatively, a balance load prediction matrix may also be supplied using load components that are expressed in "moment balance" format. Then, the "random" variation of the normal force is estimated by using the following inequality

$$
|\delta N F| \leq \frac{|\delta P M 1|+|\delta P M 2|}{b} \approx|\varphi| \cdot \frac{S\left(P M 1, R_{1}, \ldots, R_{n}\right)+S\left(P M 2, R_{1}, \ldots, R_{n}\right)}{b}
$$

where $P M 1$ is the forward pitching moment, $P M 2$ is the aft pitching moment, and $b$ is the distance between the forward and aft pitching moment gages. - The numerical evaluation of the right-hand side of Eq. (15) requires the dynamic pressure itself as input. The calculation of this quantity is briefly reviewed in the next section of the paper.

\section{E. Dynamic Pressure in Compressible Flow}

The dynamic pressure in compressible flow depends on the "tunnel conditions" that are uniquely described by the total pressure $\left(P_{T}\right)$ and the Mach number $(M)$. The connection between dynamic pressure and tunnel conditions is given by the following equation (taken from Ref. [6]):

\section{DYNAMIC PRESSURE IN COMPRESSIBLE FLOW}

$$
Q=f\left(\gamma, P_{T}, M\right)=P_{T} \cdot \frac{\gamma}{2} \cdot M^{2} \cdot\left[1+\frac{\gamma-1}{2} \cdot M^{2}\right]^{-\gamma /(\gamma-1)}
$$

Then, knowing that the ratio $\gamma$ of specific heats of air equals 1.4, Eq. (17a) can be expressed as follows:

$$
Q=f\left(P_{T}, M\right)=P_{T} \cdot \frac{7}{10} \cdot M^{2} \cdot\left[1+\frac{M^{2}}{5}\right]^{-7 / 2}
$$

At this point, an estimate of the upper bound of the drag coefficient's precision error at constant dynamic pressure can be computed as all quantities on the right-hand side of Eq. (15) are defined. The corresponding result can be visualized as a contour plot. This suggestion is described in more detail in the next section.

\section{Contour Plots of Upper Bound}

\section{A. General Remarks}

An estimate of the upper bound of the drag coefficient's precision error at constant dynamic pressure can now be computed as all quantities on the right-hand side of Eq. (15) are known. This precision error estimate is a function of (i) the given reference area $A$ of the model, (ii) an assumed angle of attack $\alpha$ of the model, (iii) the upper bound of random output variations $|\varphi|$, (iv) the partial derivatives $\partial F_{i} / \partial R_{\nu}$ of the axial and normal force of the balance, (v) the total pressure $P_{T}$, and (vi) the Mach number $M$. Therefore, after multiplying Eq. (15) by $10^{4}$ so that the upper bound of the drag coefficient's precision error is reported in "counts", Eq. (15) can be expressed as follows:

\section{PRECISION ERROR OF THE DRAG COEFFICIENT AT CONSTANT DYNAMIC PRESSURE IN COUNTS}

$$
\delta c_{D}^{\prime}=\left.10^{4} \cdot \delta c_{D}\right|_{Q=\text { const. }}=f_{1}\left(A, \alpha, \partial F_{i} / \partial R_{\nu}, M, P_{T}\right)
$$

American Institute of Aeronautics and Astronautics 
The parameter $A$ is a fixed constant for a given wind tunnel model. Similarly, the partial derivatives $\partial F_{i} / \partial R_{\nu}$ are fixed constants for a given strain-gage balance. In addition, the angle of attack $\alpha$ can be set to a constant value $\alpha_{\circ}$ that a wind tunnel user may specify. Then, the upper bound of the drag coefficient's precision error becomes exclusively a function of two variables, i.e., the Mach number and the total pressure. Consequently, the upper bound of the drag coefficient's precision error can be displayed as a 2-dimensional "contour plot" if it is plotted for a family of total pressure and Mach number combinations.

\section{B. Upper Bound versus Mach Number and Total Pressure}

In the previous section an upper bound of the drag coefficient's precision error at constant dynamic pressure was developed. In this section it will be shown how the upper bound given on the right-hand side of Eq. (12) can be visualized as a contour plot that uses the Mach number and total pressure of the wind tunnel as input. This contour plot is defined as follows:

$$
\delta c_{D}^{\prime}\left(\alpha_{\circ}\right)=f_{1}\left(\alpha_{\circ}, M, P_{T}\right)
$$

A wind tunnel model may not exceed its structural limit during a wind tunnel test. This limit is often described by the maximum dynamic pressure $Q_{\max }$ that the model can experience. Therefore, it would be helpful to also display $Q_{\max }$ in the contour plot defined in Eq. (19). A corresponding relationship involving $Q_{\max }$ can be obtained from Eq. (17a) after the equation is rearranged such that the total pressure maximum $P_{T}=P_{T \max }$ is expressed as a function of $Q_{\max }$ and $M$. Then, we get the following equation:

\section{TOTAL PRESSURE MAXIMUM OF MODEL}

$$
P_{\text {Tmax }}=Q_{\max } \cdot \frac{2}{\gamma} \cdot \frac{1}{M^{2}} \cdot\left[1+\frac{\gamma-1}{2} \cdot M^{2}\right]^{\gamma /(\gamma-1)}
$$

Again, the ratio $\gamma$ of the specific heats of air equals 1.4. Therefore, we get for air:

$$
P_{\operatorname{Tmax}}=Q_{\max } \cdot \frac{10}{7} \cdot \frac{1}{M^{2}} \cdot\left[1+\frac{\gamma-1}{2} \cdot M^{2}\right]^{7 / 2}
$$

Now, after including the total pressure maximum of the model in the contour plot, we get the following expanded contour plot of the upper bound of the drag coefficient's precision error (see also sample plots given in Figs. 6a, 8a, $8 b$ of the paper):

\section{PRECISION ERROR OF THE DRAG COEFFICIENT VERSUS MACH NUMBER AND TOTAL PRESSURE}

$$
\text { Contour Plot No. } 1 \Longrightarrow \delta c_{D}^{\prime}\left(\alpha_{\circ}\right)=f_{1}\left(\alpha_{\circ}, M, P_{T}, P_{T \max }\right)
$$

It is also possible to develop a similar contour plot of the upper bound of the drag coefficient's precision error at constant dynamic pressure if the Reynolds number instead of the total pressure is used to describe the tunnel conditions. This alternate version of the contour plot is described in the next section of the paper.

\section{Upper Bound versus Mach Number and Reynolds Number}

Some wind tunnel customers prefer to use the unit Reynolds number $R e^{\prime}$ expressed in millions per unit length instead of the total pressure $P_{T}$ for the description of the tunnel conditions. The required relationship for the unit Reynolds number can easily be derived from the general definition of the Reynolds number. The Reynolds number is defined by the following relationship (Ref. [6])

$$
R e=(\rho \cdot V \cdot l) / \mu
$$


where $\rho$ is the density, $V$ is the free-stream velocity, $l$ is a reference length, and $\mu$ is the dynamic viscosity. The unit Reynolds number in millions per unit length is obtained after dividing both sides of Eq. (22a) by the reference length $l$ and $10^{6}$. We get:

$$
R e^{\prime}=\frac{R e}{l \cdot 10^{6}}=\frac{\rho \cdot V}{\mu \cdot 10^{6}}
$$

The product of density with the free-stream velocity can be related to the dynamic pressure by using the following relationship:

$$
Q=\frac{\rho}{2} \cdot V^{2} \Longrightarrow \rho \cdot V=\frac{2 \cdot Q}{V}
$$

It is also known from the definition of the Mach number that the free-stream velocity can be expressed as a function of the Mach number and the speed of sound. Then, we get

$$
M=\frac{V}{a}=\frac{V}{\sqrt{\gamma R T}} \Longrightarrow V=M \cdot \sqrt{\gamma R T}
$$

where $\gamma$ is the ratio of the specific heats, $R$ is the gas constant, and $T$ is the static temperature in units of ${ }^{\circ} \mathrm{K}$ or ${ }^{\circ} R$. Most wind tunnels are operated by using air. Its gas constant equals $286.9\left[\mathrm{~J} /\left(\mathrm{kg}{ }^{\circ} \mathrm{K}\right)\right]$ or $1716\left[\mathrm{ft}^{2} /\left(\mathrm{sec}^{2}{ }^{\circ} R\right)\right]$. Now, after replacing the denominator on the right-hand side of Eq. (23a) with the right-hand side of Eq. (23b), the product of density with the free-stream velocity can be written as follows:

$$
\rho \cdot V=\frac{2 \cdot Q}{M \cdot \sqrt{\gamma R T}}
$$

Finally, after using the right-hand side of Eq. (23c) to replace the numerator of the right-hand side of Eq. (22b) and knowing from Eq. (17a) that the dynamic pressure $Q$ in compressible flow is a function of the ratio $\gamma$ of the specific heats, the total pressure $P_{T}$, and the Mach number $M$, the unit Reynolds number $R e^{\prime}$ in millions per unit length can be computed by using the following expression:

\section{UNIT REYNOLDS NUMBER IN MILLIONS PER UNIT LENGTH}

$$
R e^{\prime}=\frac{2 \cdot Q}{\mu \cdot M \cdot \sqrt{\gamma R T} \cdot 10^{6}}=\frac{2 \cdot f\left(\gamma, P_{T}, M\right)}{\mu \cdot M \cdot \sqrt{\gamma R T} \cdot 10^{6}}
$$

The maximum unit Reynolds number associated with the structural limit of the wind tunnel model can directly be computed by using $Q_{\max }$ and $P_{T \max }$ instead of $Q$ and $P_{T}$ in Eq. (24a) above. Then, we get:

\section{UNIT REYNOLDS NUMBER MAXIMUM OF MODEL}

$$
\operatorname{Re}_{\max }^{\prime}=\frac{2 \cdot Q_{\max }}{\mu \cdot M \cdot \sqrt{\gamma R T} \cdot 10^{6}}=\frac{2 \cdot f\left(\gamma, P_{\operatorname{Tmax}}, M\right)}{\mu \cdot M \cdot \sqrt{\gamma R T} \cdot 10^{6}}
$$

In general, the dynamic viscosity $\mu$ of a gas is a function of the static temperature. For example, the following relationship for the dynamic viscosity of air is given in the literature (see Ref. [7]):

$$
\text { air } \Longrightarrow \text { temperatures in }{ }^{\circ} \mathrm{K} \Longrightarrow \mu=1.458 \times 10^{-6} \cdot \frac{T^{3 / 2}}{T+110.4}\left[\frac{N \mathrm{sec}}{\mathrm{m}^{2}}\right]
$$

This relationship can be converted from $\left[m, N,{ }^{\circ} K\right]$ to $\left[\mathrm{ft}, \mathrm{lbf},{ }^{\circ} R\right]$ by using the following two unit conversions ( $l b f \equiv$ pound force):

$$
1.458 \times 10^{-6}\left[N \sec m^{-2}{ }^{\circ} K^{-0.5}\right] \equiv 2.270 \times 10^{-8}\left[\operatorname{lbf} \sec f t^{-2}{ }^{\circ} R^{-0.5}\right]
$$




$$
110.4\left[{ }^{\circ} K\right] \equiv 198.7\left[{ }^{\circ} R\right]
$$

Then, an alternate relationship for the dynamic viscosity is given as follows (see also Ref. [6], p. 19):

$$
\text { air } \Longrightarrow \text { temperatures in }{ }^{\circ} R \Longrightarrow \mu=2.270 \times 10^{-8} \cdot \frac{T^{3 / 2}}{T+198.7}\left[\frac{l b f \text { sec }}{f t^{2}}\right]
$$

Finally, assuming that a reasonable value for the static temperature $T$ is selected, the unit Reynolds number can be used to define a contour plot of the drag coefficient's precision error. Therefore, we can write (see also sample plot given in Fig. $6 b$ of the paper):

\section{PRECISION ERROR OF THE DRAG COEFFICIENT VERSUS MACH NUMBER AND UNIT REYNOLDS NUMBER}

$$
\text { Contour Plot No. } 2 \Longrightarrow \delta c_{D}^{\prime}\left(\alpha_{\circ}\right)=f_{2}\left(\alpha_{\circ}, M, R e^{\prime}, R e_{\max }^{\prime}\right)
$$

Figure 4 below summarizes the most important steps that are described in the paper in order to prepare contour plots of the upper bound of the drag coefficient's precision error.

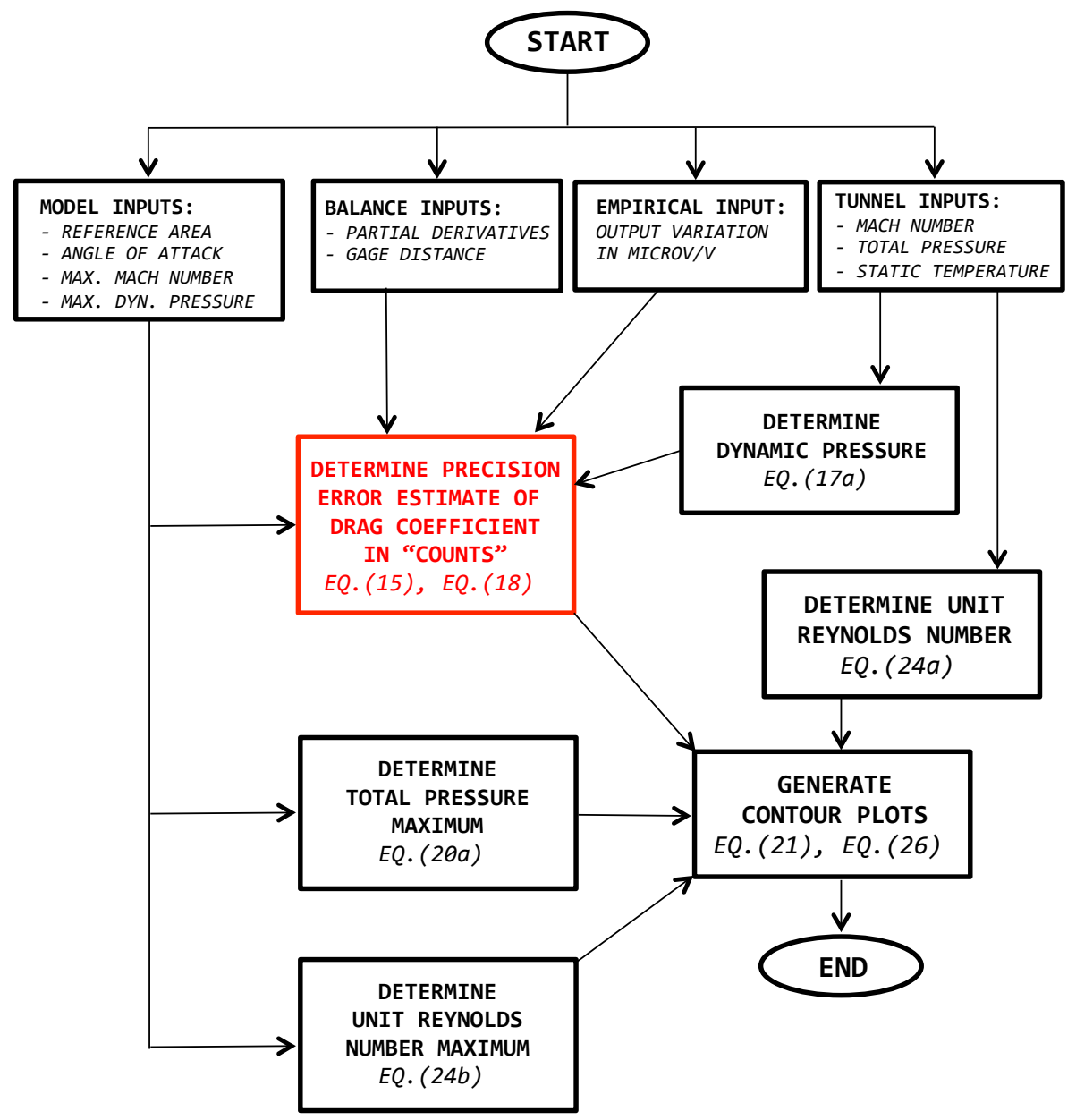

Fig. 4 Summary of the calculation of a pre-test estimate of the precision error of the drag coefficient.

The next section discusses the practical calculation of the partial derivatives of the balance load components that are needed for the assessment of small variations of the drag force measurement. Afterwards, two wind tunnel test cases are used to demonstrate the assessment of the drag coefficient's precision error. 


\section{Identification of Partial Derivatives}

It is critical for the calculation of the upper bound of the small variations of the drag force measurement that the partial derivatives of the required balance load components (e.g., axial force, normal force, forward/aft normal force, forward/aft pitching moment) with respect to the electrical outputs of the balance gages are known (see Eq. (13c)). Fortunately, least squares estimates of these partial derivatives can directly be obtained from the coefficient matrices that the Non-Iterative Method or Iterative Method use for the prediction of balance loads (see, e.g., Ref. [8] for a detailed discussion of these two methods that are traditionally used in the aerospace testing community to predict balance loads).

The Non-Iterative Method fits the balance loads directly as a function of the measured outputs of the strain-gages. Therefore, the regression models of the load components have the following general form

$$
F_{i}=\underbrace{d_{\circ}(i)}_{\text {intercept }}+\underbrace{d_{1}(i) \cdot R_{1}+\cdots+d_{n}(i) \cdot R_{n}}_{\text {linear terms }}+\cdots \quad ; \quad 1 \leq i \leq n
$$

where $F_{i}$ is the balance load with index $i, n$ is the number of loads or gage outputs of the balance, $R_{1}$ to $R_{n}$ are the independently measured outputs of the balance, and $d_{\circ}, d_{1}, \ldots$ are the regression coefficients of the regression model of the load. The regression coefficients of the linear terms of Eq. (27a) are the least squares approximations of the partial derivatives of the balance load. This observation can be described as follows:

$$
d_{\nu}(i) \approx \frac{\partial F_{i}}{\partial R_{\nu}} \quad ; \quad 1 \leq \nu \leq n
$$

The identification of approximations of the partial derivatives of the axial and normal force is also possible if an analyst uses the Iterative Method for the balance load prediction. This approach first fits the outputs as a function of the loads. The corresponding regression model of the outputs can be written as

$$
R_{i}=\underbrace{c_{0}(i)}_{\text {intercept }}+\underbrace{c_{1}(i) \cdot F_{1}+c_{2}(i) \cdot F_{2}+\cdots+c_{n}(i) \cdot F_{n}}_{\text {linear terms }}+\cdots \quad ; \quad 1 \leq i \leq n
$$

where $R_{i}$ is the measured electrical output of the gage with index $i, n$ is the number of loads or gage outputs of the balance, $F_{1}$ to $F_{n}$ are the loads of the balance, and $c_{\circ}, c_{1}, \ldots$ are the regression coefficients of the regression model of the output. Afterwards, a load iteration scheme is constructed from the regression models of the outputs so that loads can be predicted from outputs during a wind tunnel test. Two load iteration equation choices, i.e., Load Iteration Method A and Load Iteration Method B, are available if the Iterative Method is used. They can be summarized by the following two iteration equations:

$$
\begin{aligned}
& \underbrace{\text { Load Iteration Method } A}_{\text {described as Method } 1 \text { in Ref. [8] }} \Longrightarrow \mathbf{F}_{\mathbf{j}}=\underbrace{\left[\mathbf{C}_{\mathbf{1}}^{-\mathbf{\Delta}} \mathbf{\Delta}\right]}_{\text {constant }}-\underbrace{\left[\mathbf{C}_{\mathbf{1}}^{-1} \mathbf{C}_{\mathbf{2}}\right] \cdot \mathbf{H}_{\mathbf{j}-\mathbf{1}}}_{\text {changes for each iteration step }} \\
& \underbrace{\text { Load Iteration Method } B}_{\text {described as Method } 2 \text { in Ref. [8] }} \Longrightarrow \mathbf{F}_{\mathbf{j}}=\underbrace{\left[\mathbf{B}_{\mathbf{1}}^{-\mathbf{\Delta}} \boldsymbol{\Delta} \mathbf{R}\right]}_{\text {constant }}-\underbrace{\left[\mathbf{B}_{\mathbf{1}}^{-\mathbf{1}} \mathbf{B}_{\mathbf{2}}\right] \cdot \mathbf{F}_{\mathbf{j}-\mathbf{1}}-\left[\mathbf{B}_{\mathbf{1}}^{-\mathbf{1}} \mathbf{C}_{\mathbf{2}}\right] \cdot \mathbf{H}_{\mathbf{j}-\mathbf{1}}}_{\text {changes for each iteration step }}
\end{aligned}
$$

The coefficients in the rows of the two inverse matrices used above (matrix $\mathbf{C}_{\mathbf{1}}^{-\mathbf{1}}$ for Load Iteration Method $A$, or, matrix $\mathbf{B}_{1}^{-1}$ for Load Iteration Method $B$ ) have the desired approximations of the partial derivatives of the balance loads. They can directly be taken from the "Data Reduction Matrix" of the balance (see Ref. [4], p. 19, p. 62 for a definition of this matrix). It is important to mention that, by design, all off-diagonal coefficients of matrix $\mathbf{B}_{\mathbf{1}}^{-1}$ are zero. Therefore, the drag coefficient's precision error estimate obtained from the coefficients of $\mathbf{B}_{1}^{-1}$ will always be less conservative than the alternate estimate obtained from the coefficients of $\mathbf{C}_{\mathbf{1}}^{-1}$. - The magnitude of the precision error contribution associated with the normal force measurement of the balance is assessed in more detail in the next section of the paper.

\section{Precision Error Contribution of the Normal Force}

It is interesting to analyze the numerator of the fraction on the right-hand side of Eq. (15) in more detail. It can be used to quantify the size of the contribution of the normal force measurement of the balance to 
the total precision error of the predicted drag coefficient. The second product of the numerator of Eq. (15) represents the contribution of the normal force measurement to the precision error. Therefore, the ratio between this second term and the numerator of Eq. (15) itself can be used to express the contribution of the normal force measurement to the precision error as a percentage of the total precision error of the drag coefficient. Then, after simplifying the trigonometric functions of the result, we get the following expression for the percentage of the normal force contribution to the total precision error of the drag coefficient

$$
\theta=\frac{S\left(N F, R_{1}, \ldots\right) \cdot \tan |\alpha|}{S\left(A F, R_{1}, \ldots\right)+S\left(N F, R_{1}, \ldots\right) \cdot \tan |\alpha|} \times 100 \%
$$

where the upper bounds $S\left(N F, R_{1}, \ldots\right)$ and $S\left(A F, R_{1}, \ldots\right)$ are obtained after replacing $F_{i}$ in Eq. (13c) by either $N F$ or $A F$. The upper bound of the partial derivatives of the normal force can easily be obtained from coefficients of the related load prediction matrix if the loads are given in direct-read format. Alternate definitions have to be used if loads and the associated load prediction matrix are given in either force or moment balance format. Then, the following upper bounds should be used (see also Eqs. (16a) and (16b)):

$$
\begin{aligned}
\text { force balance format } & \Longrightarrow S\left(N F, R_{1}, \ldots\right) \approx S\left(N 1, R_{1}, \ldots\right)+S\left(N 2, R_{1}, \ldots\right) \\
\text { moment balance format } & \Longrightarrow S\left(N F, R_{1}, \ldots\right) \approx\left[S\left(P M 1, R_{1}, \ldots\right)+S\left(P M 2, R_{1}, \ldots\right)\right] / b
\end{aligned}
$$

The percentage $\theta$ can easily be evaluated for a strain-gage balance if (i) calibration analysis results are available and (ii) a reasonable family of angle of attack values is selected. Data from NASA's ARC30K 5component semi-span balance can be used to illustrate the calculation of the percentage $\theta$ because results of the calibration analysis for the Iterative Method were available (see Ref. [8] for a description of the Iterative Method). Table 1 below lists all sensitivity estimates of gage set 1 that were obtained from Triumph Aerospace's 2013 calibration of the balance.

Table 1: $\left[\partial R_{i} / \partial F_{j}\right] \equiv$ sensitivities of the ARC30K semi-span balance. ${ }^{\dagger}$

\begin{tabular}{|c|c|c|c|c|c|}
\hline & $R_{1}=r N F$ & $R_{2}=r A F$ & $R_{3}=r P M$ & $R_{4}=r Y M$ & $R_{5}=r R M$ \\
\hline \hline$F_{1}=N F$ & $+\mathbf{0 . 0 4 0 4 0 7}$ & -0.000473 & +0.000043 & +0.000000 & +0.001497 \\
\hline$F_{2}=A F$ & -0.000076 & $\mathbf{+ 0 . 3 9 6 8 6 5}$ & -0.000206 & +0.002971 & +0.000072 \\
\hline$F_{3}=P M$ & +0.000112 & -0.000007 & $+\mathbf{0 . 0 0 5 4 9 3}$ & -0.000018 & -0.000010 \\
\hline$F_{4}=Y M$ & +0.000010 & -0.000016 & +0.000008 & $+\mathbf{0 . 0 0 2 0 4 3}$ & -0.000001 \\
\hline$F_{5}=R M$ & +0.000029 & -0.000002 & -0.000003 & +0.000004 & $+\mathbf{0 . 0 0 1 0 5 7}$ \\
\hline
\end{tabular}

${ }^{\dagger}$ The sensitivities are the coefficients of matrix $\mathbf{C}_{\mathbf{1}} ;$ unit $\equiv(\mu V / V) / l b f$ or $(\mu V / V) /($ in-lbf $)$.

The sensitivities reported in Table 1 above are the coefficients of matrix $\mathbf{C}_{\mathbf{1}}$ that results from the least squares fit of the five primary bridge outputs of the ARC30K balance. The partial derivatives $\partial F_{i} / \partial R_{j}$ required for the calculation of $S\left(N F, R_{1}, \ldots\right)$ and $S\left(A F, R_{1}, \ldots\right)$ in Eq. (29a) can immediately be obtained by computing the inverse of matrix $\mathbf{C}_{\mathbf{1}}$. Then, we get:

Table 2: $\left[\partial F_{i} / \partial R_{j}\right] \equiv$ partial derivatives of the loads of the ARC30K semi-span balance. ${ }^{\dagger}$

\begin{tabular}{|c|c|c|c|c|c|}
\hline & $R_{1}=r N F$ & $R_{2}=r A F$ & $R_{3}=r P M$ & $R_{4}=r Y M$ & $R_{5}=r R M$ \\
\hline \hline$F_{1}=N F$ & $+\mathbf{2 4 . 7 7 3 7 6}$ & +0.005544 & -0.505730 & -0.123317 & -0.671750 \\
\hline$F_{2}=A F$ & +0.029365 & $\mathbf{+ 2 . 5 1 9 6 1 6}$ & +0.002770 & +0.018963 & +0.002795 \\
\hline$F_{3}=P M$ & -0.213838 & +0.099419 & $\mathbf{+ 1 8 2 . 0 4 3 0}$ & -0.684369 & +0.594870 \\
\hline$F_{4}=Y M$ & +0.018637 & -3.663806 & +1.593760 & $+\mathbf{4 8 9 . 5 4 6 6}$ & -1.705461 \\
\hline$F_{5}=R M$ & -35.08594 & -0.182163 & +2.491116 & +0.533006 & $\mathbf{+ 9 4 6 . 9 6 5 2}$ \\
\hline
\end{tabular}

${ }^{\dagger}$ The partial derivatives are the coefficients of matrix $\mathbf{C}_{\mathbf{1}}^{-1}$; unit $\equiv l b f /(\mu V / V)$ or $($ in-lbf $) /(\mu V / V)$. 
Now, all information needed for the calculation of the percentage $\theta$ is available. First, the upper bounds of the partial derivatives of the normal and axial force of the balance are computed by using the definition that is given in Eq. (13c). The following results can be obtained by using the contents of Table 2 :

$$
\begin{aligned}
& S\left(N F, R_{1}, \ldots, R_{5}\right)=\sqrt{\sum_{\nu=1}^{5}\left[\frac{\partial N F}{\partial R_{\nu}}\right]^{2}} \approx 25 \mathrm{lbf} /(\mu V / V) \\
& S\left(A F, R_{1}, \ldots, R_{5}\right)=\sqrt{\sum_{\nu=1}^{5}\left[\frac{\partial A F}{\partial R_{\nu}}\right]^{2}} \approx 2.5 \mathrm{lbf} /(\mu V / V)
\end{aligned}
$$

Finally, a family of angles of attack is chosen and so that the normal force contribution percentage defined in Eq. (29a) can be computed. We get the following results:

Table 3: Normal force contribution to the total precision error of the drag coefficient.

\begin{tabular}{|c|c|c|c|c|c|c|}
\hline & $\alpha=0^{\circ}$ & $\alpha=2^{\circ}$ & $\alpha=4^{\circ}$ & $\alpha=6^{\circ}$ & $\alpha=8^{\circ}$ & $\alpha=10^{\circ}$ \\
\hline \hline$\theta$ & $0 \%$ & $26 \%$ & $41 \%$ & $51 \%$ & $58 \%$ & $64 \%$ \\
\hline
\end{tabular}

It can be concluded from Table 3 that the contribution of the normal force to the total precision error of the drag coefficient must not be underestimated if the primary sensitivities of the normal and axial force gages differ by one order of magnitude. This situation applies to the ARC30K balance because we know from Table 1 that the primary sensitivity $\partial(r N F) / \partial(N F)$ of the normal force gage equals about 1/10 of the primary sensitivity $\partial(r A F) / \partial(A F)$ of the axial force gage. In that case, the contribution of the normal force to the total precision error of the drag coefficient will be $51 \%$, i.e., it exceeds the contribution of the axial force, if the angle of attack is near 6 degrees. By design, most balances used for both performance and stability \& control tests of a traditional aircraft have axial and normal force sensitivities that differ by one order of magnitude. Therefore, an increase of the normal force sensitivity requirement for a new balance design can significantly decrease the precision error of the drag coefficient. Such a sensitivity increase could be achieved by specifically designing and fabricating a balance with reduced normal force capacity for the performance portion of the wind tunnel test. - Results of the application of the pre-test assessment of the drag coefficient's precision error to two wind tunnel test situations are presented in the next section.

\section{Discussion of Example}

Two examples are discussed in this section in order to illustrate results of the assessment of the drag coefficient's precision error for typical wind tunnel test situations. The first example consist of the HATOL model that was tested in 2015 in the NASA Ames 11-ft TWT (the model is shown in Fig. 5 below).

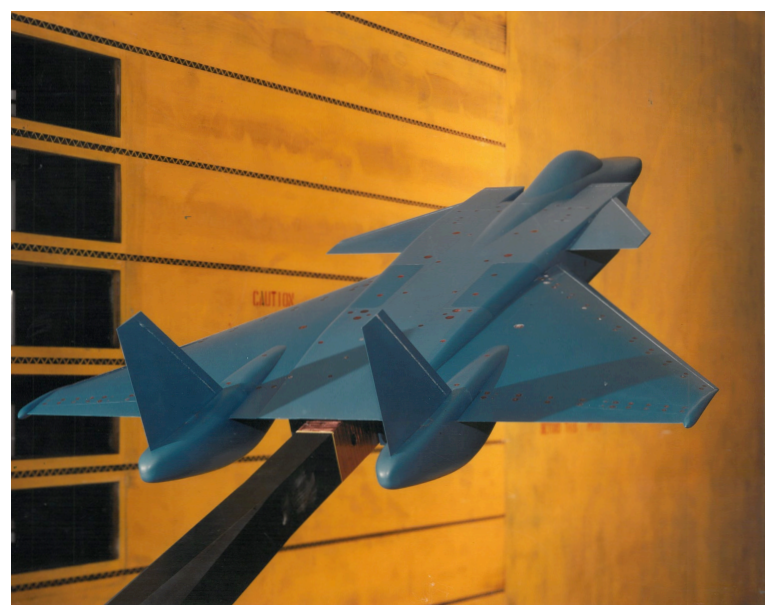

Fig. 5 HATOL model installed in the test section of the NASA Ames 11-ft Transonic Wind Tunnel (photo courtesy of Don Durston, NASA Ames Research Center). 
The HATOL model is a low aspect ratio generic supersonic fighter model. Loads were measured during the test by using the 2.0 inch MK29A six-component force balance. It was decided to estimate the drag coefficient's precision error for this test situation by assuming that the model's angle of attack $\alpha_{\circ}$ is fixed at zero degrees. Then, Eq. (15) was used to compute the precision error of the drag coefficient at constant dynamic pressure. Figure 6a shows the drag coefficient's precision error in "counts" plotted as a function of the Mach number and total pressure.

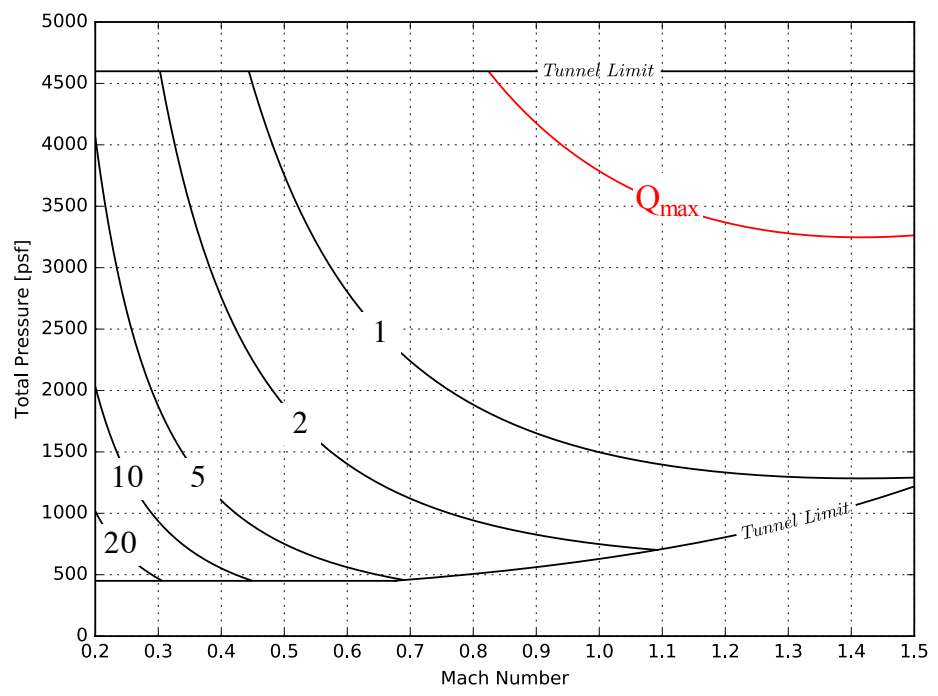

Fig. 6a Precision error of the drag coefficient at constant dynamic pressure plotted in "counts" versus Mach number \& total pressure (HATOL, MK29A, A =4.5 ft $\mathrm{ft}^{2} \alpha_{\circ}=0^{\circ}, Q_{\max }=1400 \mathrm{psf}$ ).

Similarly, Fig. $6 b$ shows the drag coefficient's precision error in "counts" plotted as a function of the Mach number and unit Reynolds number.

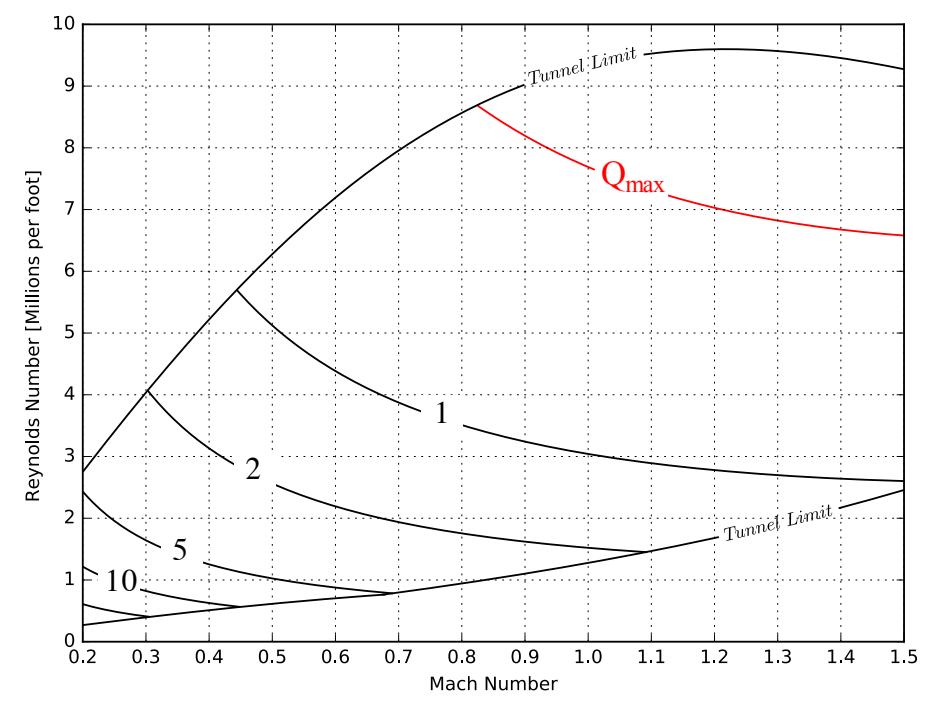

Fig. $6 b$ Precision error of the drag coefficient at constant dynamic pressure plotted in "counts" versus Mach number \& Reynolds number (HATOL, MK29A, A=4.5 $\mathrm{ft}^{2}, \alpha_{\circ}=0^{\circ}, Q_{\max }=1400 \mathrm{psf}$ ).

Both plots clearly highlight the fact that smaller precision errors can be achieved at higher Mach numbers and total pressures. - The second example assesses the drag coefficient's precision error of the UHB semispan model assuming it is used in combination with either NASA's MC400 or ARC30K semi-span balance for a test in the NASA Ames 11-ft TWT. The UHB model is a generic high aspect ratio subsonic commercial transport type wind tunnel model (see Fig. 7a below). 


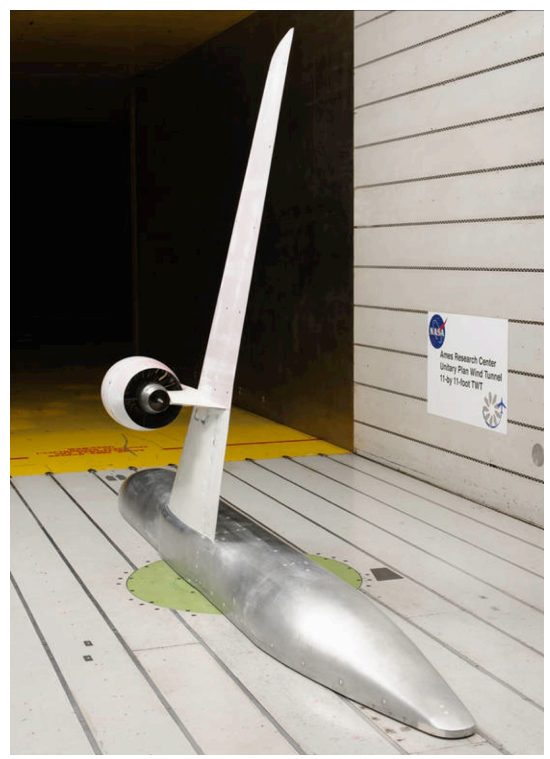

Fig. 7a UHB model installed in the test section of the NASA Ames 11-ft Transonic Wind Tunnel (photo courtesy of the Wind Tunnel Operations Division, NASA Ames Research Center).

The two balances are shown in Fig. $7 b$ below. The MC400 balance was originally designed for the NASA Ames 12-ft Pressure Wind Tunnel. This wind tunnel had a maximum total pressure of $6 \mathrm{~atm}$. The Ames 11-ft TWT, on the other hand, has a maximum total pressure of $2 \mathrm{~atm}$. Therefore, the capacities of
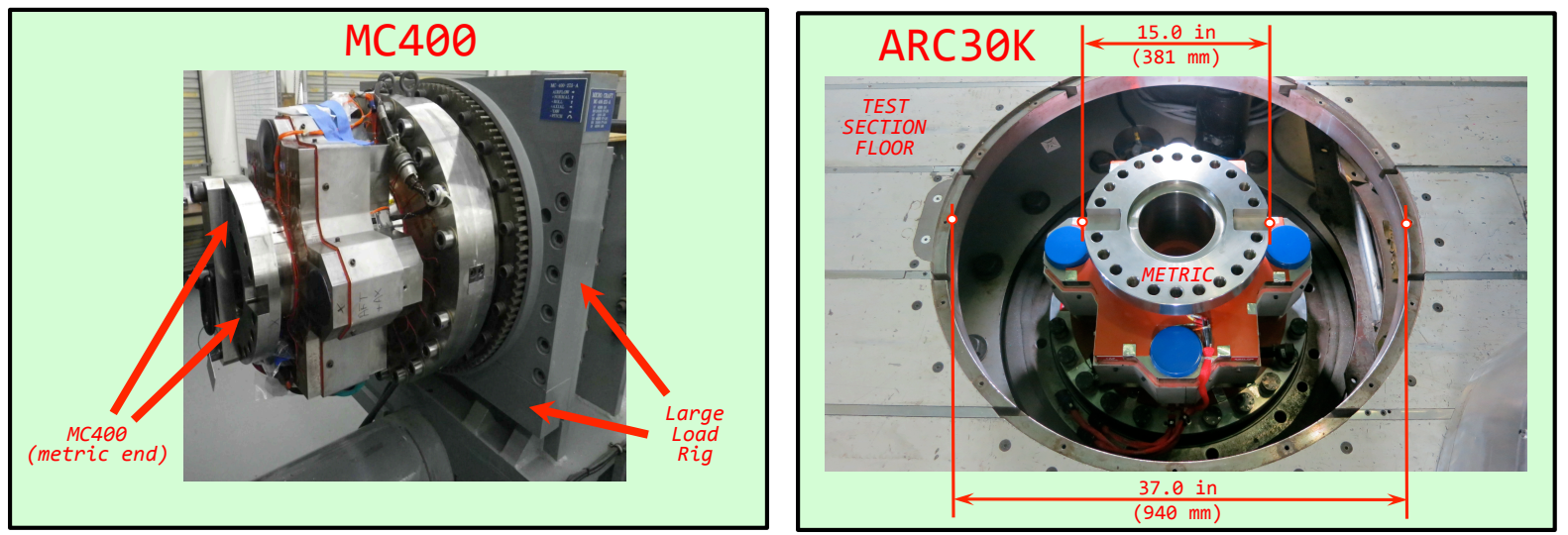

Fig. '7b NASA's MC400 and ARC30K high capacity 5-component semi-span balances.

the MC400 are much larger than the expected loads for a typical semi-span model that is tested in the Ames 11-ft TWT. In other words, the MC400 is not ideally sized for semi-span model tests in the 11-ft TWT. The ARC30K balance, of the other hand, was specifically designed for use in the Ames 11-ft TWT. Consequently, it has lower capacities and higher sensitivities when compared with the MC400 (see Table 4).

Table 4: Normal and axial force capacities and sensitivities of two NASA semi-span balances.

\begin{tabular}{|c|c|c|c|c|}
\hline & $N F_{\max }$ & $A F_{\max }$ & $\partial(r N F) / \partial(N F)$ & $\partial(r A F) / \partial(A F)$ \\
\hline \hline MC400 & $40,000[l b f]$ & $8,000[l b f]$ & $0.0223[(\mu V / V) / l b f]$ & $0.0795[(\mu V / V) / l b f]$ \\
\hline ARC30K & $30,000[l b f]$ & $3,000[l b f]$ & $0.0404[(\mu V / V) / l b f]$ & $0.3969[(\mu V / V) / l b f]$ \\
\hline
\end{tabular}

Again, it was decided to estimate the drag coefficient's precision error for the given wind tunnel test situation by assuming that the model's angle of attack $\alpha_{\circ}$ is fixed at zero degrees. Then, Eq. (15) was used to compute the precision error at constant dynamic pressure. Figure $8 a$ below depicts the drag coefficient's 
precision error estimate in "counts" plotted as a function of the Mach number and total pressure if the MC400 balance would be selected for the wind tunnel test.

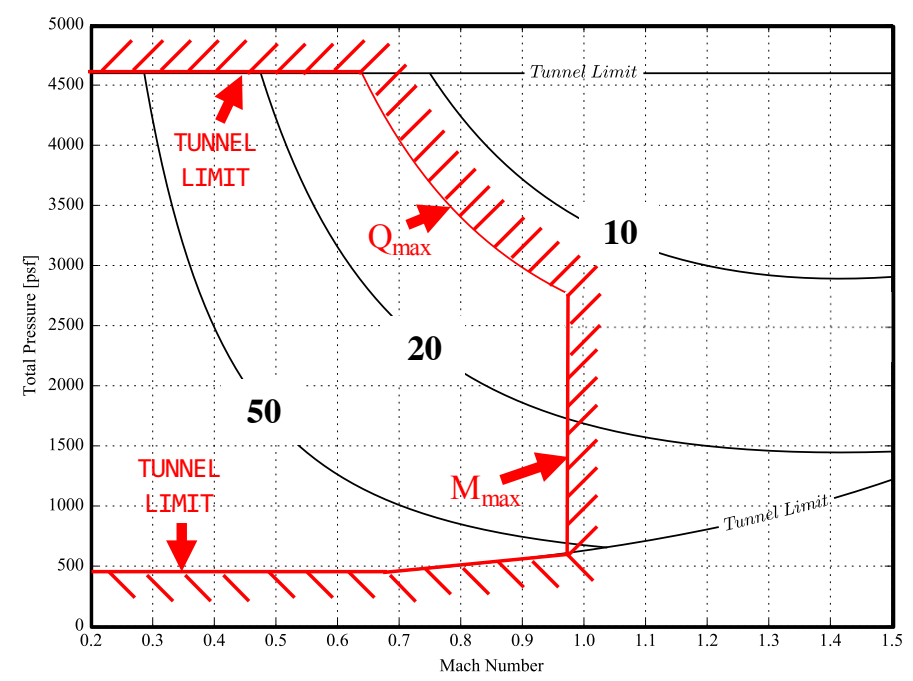

Fig. 8 a $M C 400 \Longrightarrow$ Precision error of the drag coefficient at constant dynamic pressure plotted in "counts" versus the Mach number \& total pressure (UHB, $\left.A=10.1 \mathrm{ft}^{2}, \alpha_{\circ}=0^{\circ}, Q_{\max }=1000 \mathrm{psf}\right)$.

The hash marks shown in Fig. 8a identify the boundaries of the test envelope of the model. The boundaries are defined by the tunnel limit, and, the dynamic pressure \& Mach number limits (i.e., $Q_{\max }$ and $M_{\max }$ ) of the model itself. The contour plot also confirms that the overall precision error of the drag coefficient can be reduced if tests are conducted at higher Mach numbers and total pressures. Figure $8 b$ shows the

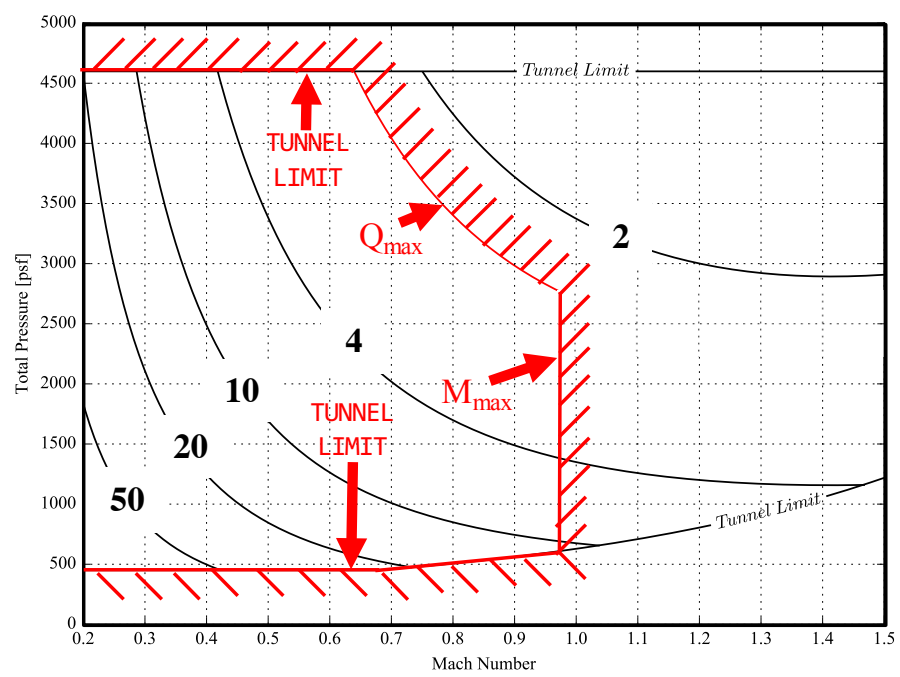

Fig. $8 b$ ARC30K $\Longrightarrow$ Precision error of the drag coefficient at constant dynamic pressure plotted in "counts" versus the Mach number $\& 5$ total pressure (UHB, $\left.A=10.1 \mathrm{ft}^{2}, \alpha_{\circ}=0^{\circ}, Q_{\max }=1000 \mathrm{psf}\right)$.

precision error of the drag coefficient for the ARC30K balance. Significantly lower precision errors can be expected for the ARC30K balance because it has substantially higher normal and axial force sensitivities when compared with the MC400. The ARC30K balance is clearly the better choice for the wind tunnel test.

\section{Summary and Conclusions}

A new method was presented that computes a realistic pre-test estimate of the upper bound of the precision error of a wind tunnel model's drag coefficient. The calculation of the upper bound only requires some basic information about the model, the balance, the tunnel conditions, and an "empirical" limit of the 
expected gage output variations as input. Results of the precision error assessment are reported in contour plots that depict the precision error for a fixed angle of attack as a function of the Mach number and total pressure (or unit Reynolds number). Data from two wind tunnel models and three balances were used to illustrate the calculation of the upper bound of the drag coefficient's precision error.

Precision error contributions at constant dynamic pressure and at constant drag were investigated independently. The chosen approach made it possible to clearly separate the influence of errors associated with the measurement of the aerodynamic loads (i.e., with the measurement of balance loads and angle of attack) from errors associated with the measurement of the tunnel conditions (i.e., with the measurement of the dynamic pressure). This basic idea can also be used to obtain pre-test precision error estimates for other aerodynamic coefficients like, e.g., the lift coefficient or the pitching moment coefficient, because their definitions and total differentials all follow the same basic pattern (see App. 4 for more details).

The rigorous analysis of the drag coefficient's precision error clearly shows that the precision error contribution associated with the measurement of the aerodynamic loads (i.e., the precision error at constant dynamic pressure) is often one order of magnitude larger than the precision error contribution associated with the measurement of the tunnel conditions (i.e., the precision error at constant drag). Therefore, a first conclusion can be drawn that is valid for most wind tunnel test situations:

\section{CONCLUSION 1}

The contribution of balance load \& angle of attack measurements to the total precision error of the drag coefficient is substantially larger than the contribution of the dynamic pressure measurement.

Furthermore, knowing from Eq. (15) that the precision error of the drag coefficient at constant dynamic pressure is directly proportional to the inverse of the dynamic pressure, the following second conclusion can be drawn:

\section{CONCLUSION 2}

The drag coefficient's precision error reaches its minimum for a given test configuration and angle of attack if the model is tested at the maximum dynamic pressure that its structural load limit permits.

In addition, it was shown in the paper that the sensitivities of the normal and axial force gages of the balance dictate the overall magnitude of the precision error of the drag coefficient as the error contribution associated with the angle of attack measurement is "hidden" in the electrical output variations of the normal and axial force gages. Consequently, a third conclusion can be drawn:

\section{CONCLUSION 3}

The larger the sensitivities of the normal and axial force gages of the balance are, the smaller the precision error of the predicted drag coefficient will be.

The investigations also demonstrated that the normal force sensitivity of a balance can have a surprisingly strong influence on the overall magnitude of the drag coefficient's precision error. A typical balance chosen for tests of commercial transport type aircraft is often sized such that it can be used during both the performance and stability \& control portion of the planned wind tunnel test. This balance design characteristic, however, directly translates into a normal force sensitivity of the balance that is about one order of magnitude lower than its axial force sensitivity. In that case, two balance force measurements of substantially different resolution are combined when the drag coefficient is computed. Consequently, assuming that the wind tunnel model is tested in the neighborhood of its cruise angle of attack (i.e., between 3 and 5 degrees), the contribution of the normal force measurement to the total precision error of the drag coefficient can equal or even exceed the contribution of the axial force measurement. Therefore, the ratio between the axial and normal force sensitivities of the balance should be as close as possible to the "ideal" value of one if the precision error of the predicted drag coefficient is to be minimized. 
In principle, two modifications of the wind tunnel test configuration can be made if a wind tunnel user wants to minimize the unwanted contribution of the normal force measurement to the total precision error of the drag coefficient. Modification 1: A dedicated "reduced-capacity" balance is designed for the performance portion of the wind tunnel test that has a higher normal force sensitivity. The authors recommend to use $50 \%$ of the normal force capacity needed for the stability \& control portion of the wind tunnel test as a realistic design goal for the dedicated "reduced-capacity" balance. Modification 2: The wind tunnel model is designed such that an existing balance can be installed in the model at an inclination angle relative to the model's centerline that equals the negative of the expected cruise angle of attack of the model. Then, the influence of the normal force measurement on the total precision error of the drag coefficient reaches a minimum when the model is tested in the neighborhood of its cruise angle of attack. The suggested two modifications would, of course, increase the overall cost and/or complexity of the wind tunnel test. However, they may have to be made if a wind tunnel user requires a very low precision error of the drag coefficient (e.g., 1/2 drag count or less) to objectively identify benefits of a model configuration change.

\section{Acknowledgements}

The authors want to thank Mike Treece, Don Durston, James Bell, and Jon Bader of NASA for their critical and constructive review of the final manuscript. The work reported in this paper was supported by the Wind Tunnel Division at NASA Ames Research Center under contract NNA16BD26C.

\section{References}

${ }^{1}$ Schlichting, H., and Truckenbrodt, E., Aerodynamics of the Airplane, translated by H. J. Ramm, McGraw-Hill, Inc., 1979, p. 15.

${ }^{2}$ Courant, R., Differential and Integral Calculus - Volume II, translated by E. J. McShane, Interscience Publishers Inc. New York, 1936, p. 66.

${ }^{3}$ Ulbrich, N., and Boone, A. R., "Uncertainty Analysis of Test Data in the Ames 11-Foot Transonic Wind Tunnel," AIAA 2004-2195, paper presented at the 24th AIAA Aerodynamic Measurement Technology and Ground Testing Conference, Portland, Oregon, June/July 2004.

${ }^{4}$ AIAA/GTTC Internal Balance Technology Working Group, Recommended Practice, Calibration and Use of Internal Strain-Gage Balances with Application to Wind Tunnel Testing, AIAA R-091-2003, American Institute of Aeronautics and Astronautics, Reston, Virginia, 2003, pp. 4-5, p. 19, p. 62.

${ }^{5}$ Ulbrich, N., and Bader, J., "Analysis of Sting Balance Calibration Data Using Optimized Regression Models," AIAA 2009-5372, paper presented at the 45th AIAA/ASME/SAE/ASEE Joint Propulsion Conference, Denver, Colorado, August 2009, pp. 18-21.

${ }^{6}$ Ames Research Staff, Equations, Tables, and Charts for Compressible Flow, NACA Report 1135, Ames Aeronautical Laboratory, Moffett Field, California, 1953.

${ }^{7}$ Hilsenrath, J., et al., Tables of Thermal Properties of Gases, National Bureau of Standards Circular 564, published by the United States Department of Commerce, 1955, p. 10, p. 69.

${ }^{8}$ Ulbrich, N., "Comparison of Iterative and Non-Iterative Strain-Gage Balance Load Calculation Methods," AIAA 2010-4202, paper presented at the 27th AIAA Aerodynamic Measurement Technology and Ground Testing Conference, Chicago, Illinois, June/July 2010.

${ }^{9}$ Jackson, P., et al., Jane's All The World's Aircraft 2003-2004, 94th year of issue, Jane's Information Group, 2003, p. 214, p. 568/569.

${ }^{10}$ Jackson, P., et al., Jane's All The World's Aircraft 2000-2001, 91st year of issue, Jane's Information Group, 2000, p. 633.

${ }^{11}$ Filippone, A., "Data and Performances of Selected Aircraft and Rotorcraft," Progress in Aerospace Sciences, Vol. 36, published by Elsevier Science Ltd., 2000, p. 635, Fig. 5.

${ }^{12}$ Heffley, R. K., and Jewell, W. F., Aircraft Handling Qualities Data, NASA CR-2144, prepared by Systems Technology, Inc., Hawthorne, California, December 1972, p. 198. 


\section{Appendix 1: Aircraft Payload and Aerodynamic Drag}

In general, the owner/operator of a subsonic commercial transport aircraft is interested in maximizing the aircraft's payload or range when operated in cruise condition. Consequently, the primary goal of the performance test of a subsonic commercial transport aircraft in a wind tunnel is often the minimization of the aerodynamic drag in cruise condition as a drag reduction will directly result in an increase of the aircraft's payload or range as long as the estimated structural weight of the airframe remains unchanged.

A better understanding of the required "precision" of the drag prediction during performance tests of a subsonic commercial transport aircraft can be obtained if the connection between the aircraft's payload and the drag coefficient is quantified. The derivation of such a relationship starts with the definition of the drag coefficient. It is defined by the following relationship

$$
c_{D}=D /(Q \cdot A)
$$

where $D$ is the aerodynamic drag force, $Q$ is the dynamic pressure, and $A$ is the aircraft's reference area. It is also known from the literature that the lift-to-drag ratio $L / D$ of a subsonic commercial transport aircraft operated in cruise condition is between 17 and 19 (see Ref. [11]). Therefore, the following approximate value of the lift-to-drag ratio can be introduced for a typical subsonic commercial transport aircraft:

$$
L / D \approx 18
$$

Then, after solving the above equation for the drag force, we get the following relationship:

$$
D \approx L / 18
$$

It is also known that the forces on an aircraft in cruise condition are in equilibrium. Therefore, the magnitude of the lift must equal the weight of the aircraft. Consequently, we get the following relationship:

$$
L=W
$$

Now, after replacing the lift by the weight in Eq. (32), we get the following relationship for the drag force in cruise condition:

$$
D \approx W / 18
$$

In the next step, the drag force on the right-hand side of Eq. (30) is replaced by the right-hand side of Eq. (34). Then, we get:

$$
c_{D} \approx W /(18 \cdot Q \cdot A)
$$

Now, after rearranging Eq. (35), the aircraft's weight can be approximated by the following expression:

$$
W \approx 18 \cdot Q \cdot A \cdot c_{D}
$$

It is useful for the current investigation to have an approximate relationship between aircraft weight change and drag coefficient change. This relationship can be obtained by computing the first derivative of the total weight with respect to the drag coefficient. Then, we get:

$$
\Delta W \approx \frac{\partial W}{\partial c_{D}} \cdot \Delta c_{D}
$$

The partial derivative of the total weight with respect to the drag coefficient is obtained from Eq. (36). We get the following relationship:

$$
\frac{\partial W}{\partial c_{D}} \approx 18 \cdot Q \cdot A
$$

Then, after using the right-hand side of Eq. (38) in order to replace the partial derivative on the right-hand side of Eq. (37), we get for the aircraft weight change the following expression:

$$
\Delta W \approx 18 \cdot Q \cdot A \cdot \Delta c_{D}
$$


The payload of the aircraft still needs to be introduced in Eq. (39) so that a connection between drag coefficient change and the payload change can be established. Data of existing subsonic commercial transport aircraft may be used in order to express the payload $\left(W^{\prime}\right)$ as a multiple of the total weight $(W)$ of the aircraft at take-off. The total weight at take-off is selected for the current investigations because (i) it can easily be obtained for different subsonic commercial transport aircraft from the literature and (ii) the initial weight at cruise condition is close to the total weight at take-off. Table 5 below lists total take-off weight and payload data for three existing aircraft designs.

Table 5: Weight and payload estimates for existing aircraft $\left(g=9.81\left[\mathrm{~m} / \mathrm{sec}^{2}\right]\right)$.

\begin{tabular}{|c|c|c|c|}
\hline Aircraft Make/Model & $W / g$ & $W^{\prime} / g$ & $W / W^{\prime}$ \\
\hline \hline Boeing B747-400F & $396,895[\mathrm{~kg}] \equiv 875,000[\mathrm{lbs}]$ & $113,000[\mathrm{~kg}] \equiv 249,125[\mathrm{lbs}]$ & 3.51 \\
\hline Boeing MD11F & $273,314[\mathrm{~kg}] \equiv 602,555[\mathrm{lbs}]$ & $90,787[\mathrm{~kg}] \equiv 200,151[\mathrm{lbs}]$ & 3.01 \\
\hline Airbus A380F & $590,000[\mathrm{~kg}] \equiv 1,300,720[\mathrm{lbs}]$ & $150,000[\mathrm{~kg}] \equiv 330,700[\mathrm{lbs}]$ & 3.93 \\
\hline
\end{tabular}

${ }^{\dagger}$ weight \& payload taken from Ref. [9] ; ${ }^{\ddagger}$ weight \& payload taken from Ref. [10] ; lbs $\equiv$ pound mass

Data given in Table 5 indicates that the ratio between take-off weight and payload of a subsonic commercial transport aircraft is between 3.01 and 3.93. It is sufficient for the current investigation to use an approximate average value of the ratios listed in Table 5. Then, we get the following estimate:

$$
\frac{W}{W^{\prime}} \approx 3.5
$$

Then, after solving Eq. (40) for the aircraft's payload, we get the following relationship:

$$
W^{\prime} \approx \frac{W}{3.5}
$$

In the next step, after replacing the aircraft weight in Eq. (41) by the right-hand side of Eq. (36), we get the following relationship between the payload and the drag coefficient in cruise condition:

$$
W^{\prime} \approx \frac{18 \cdot Q \cdot A \cdot c_{D}}{3.5}
$$

Finally, the desired relationship between payload change and drag coefficient change can be obtained after dividing both sides of Eq. (39) by both sides of Eq. (42). Then, after further simplifying the result, we get the following relationship:

\section{RELATIONSHIP BETWEEN PAYLOAD AND DRAG COEFFICIENT CHANGE}

$$
\frac{\Delta W}{W^{\prime}} \approx \frac{3.5}{c_{D}} \cdot \Delta c_{D}
$$

The right-hand side of Eq. (43) can be interpreted as the aircraft's weight change per unit payload that is associated with a given drag coefficient change. A realistic estimate for the right-hand side of Eq. (43) can immediately be obtained if a reasonable estimate of the drag coefficient $c_{D}$ in cruise condition is made. This estimate can be computed by applying Eq. (35). The take-off weight $W$ and wing reference area $A$ for different subsonic commercial transport aircraft can easily be obtained from the literature (see Table 6 below). An estimate of the dynamic pressure in cruise condition is also needed. In general, the dynamic pressure in compressible flow is given by the following equation

$$
Q=\frac{\gamma}{2} \cdot p^{*} \cdot M^{2}
$$


where the ratio $\gamma$ of the specific heats of air equals 1.4. In addition, assuming that the cruising altitude is $10,000[\mathrm{~m}]$ or 32,808 [ft], we get the following static pressure from Ref. [1]:

$$
p^{*}=26,496[\mathrm{~Pa}] \equiv 3.8429[\mathrm{psi}]
$$

It is also assumed that the cruising Mach number is 0.85 . Then, we get the following value for the dynamic pressure in cruise condition:

$$
Q=\frac{1.4}{2} \cdot 26,496 \cdot 0.85^{2}=13,400[\mathrm{~Pa}] \equiv 1.9435[\mathrm{psi}]
$$

Table 6 below lists take-off weights, reference areas, and cruise drag coefficient estimates that were computed for the chosen cruise conditions.

Table 6: Cruise drag coefficient estimate for existing aircraft $\left(g=9.81\left[\mathrm{~m} / \mathrm{sec}^{2}\right]\right)$.

\begin{tabular}{|c|c|c|c|c|}
\hline Aircraft Make/Model & $W / g$ & $W$ & $A$ & Eq. $(35) \Longrightarrow c_{D}$ \\
\hline \hline Boeing B747-400F & $396,895[\mathrm{~kg}]$ & $3,893,540[\mathrm{~N}]$ & $541\left[\mathrm{~m}^{2}\right]$ & 0.0298 \\
\hline Boeing MD11F & $273,314[\mathrm{~kg}]$ & $2,681,210[\mathrm{~N}]$ & $339\left[\mathrm{~m}^{2}\right]$ & 0.0328 \\
\hline Airbus A380F & $590,000[\mathrm{~kg}]$ & $5,787,900[N]$ & $845\left[\mathrm{~m}^{2}\right]$ & 0.0284 \\
\hline
\end{tabular}

${ }^{\dagger}$ weight \& area taken from Ref. [9] ; ${ }^{\ddagger}$ weight \& area taken from Ref. [10]

It is sufficient for the current investigation to use the average of the predicted drag coefficients of the three aircraft listed in Table 6 because the value is only used to estimate the overall magnitude of the influence of a drag coefficient change on the aircraft's payload. We get the following arithmetic mean of the drag coefficients:

$$
c_{D} \approx 0.0303
$$

Finally, a drag coefficient change needs to be selected. The following choice is made:

$$
\Delta c_{D}=0.0001 \equiv \text { one drag count }
$$

At this point, a numerical value of the expected payload change can be obtained as all quantities on the right-hand side of Eq. (43) are known. We get the following value:

$$
\frac{\Delta W}{W^{\prime}} \approx \frac{3.5}{0.0303} \cdot 0.0001 \approx 0.0116 \approx 1 \% \text { payload }
$$

The numerical result given in Eq. (47) can be summarized by the following statement if it is assumed that drag coefficient measurements during the wind tunnel test of a subsonic commercial transport aircraft in cruise condition can objectively detect a drag coefficient reduction of one count:

\section{A drag coefficient reduction of 1 count translates into about a $1 \%$ payload increase} for a typical subsonic commercial transport aircraft that operates in cruise condition. 


\section{Appendix 2: Drag Prediction Precision and Mach Number Measurement}

In Appendix 1 is was rigorously shown that an objectively observed drag coefficient change of one count, i.e., $\Delta c_{D}=0.0001$, during the wind tunnel test of a subsonic commercial transport aircraft in cruise condition can have a noticeable impact on the prediction of the aircraft's payload. Therefore, the influence of different wind tunnel measurements on the drag coefficient calculation has to be well understood so that the drag coefficient prediction error can be kept near one count whenever possible.

The precision of the Mach number measurement during a wind tunnel test is one input that influences the total drag coefficient prediction error. Therefore, it is useful to develop a relationship that directly relates an assumed small Mach number change to a resulting small drag coefficient change because this relationship can be used to relate a known Mach number uncertainty to a resulting drag coefficient prediction uncertainty. The derivation of such a relationship starts with the definition of the drag coefficient. It is defined as follows

$$
c_{D}=D /(Q \cdot A)
$$

where $D$ is the aerodynamic drag force, $Q$ is the dynamic pressure, and $A$ is the aircraft's reference area. The lift-to-drag ratio $L / D$ of a subsonic commercial transport aircraft in cruise condition is between 17 and 19 (see Ref. [11]). Therefore, the following approximate value of the lift-to-drag ratio can be introduced:

$$
L / D \approx 18
$$

Now, after solving the above equation for the drag force, we get the following relationship:

$$
D \approx L / 18
$$

It is also known that the forces on an aircraft in cruise condition are in equilibrium. Therefore, the magnitude of the lift must equal the weight of the aircraft. Consequently, we get the following relationship:

$$
L=W
$$

Now, after replacing the lift by the weight in Eq. (50), we get the following relationship for the drag force in cruise condition:

$$
D \approx W / 18
$$

In the next step, the Mach number needs to be introduced in Eq. (48). The Mach number is an independent variable of the dynamic pressure in compressible flow. The corresponding relationship for the dynamic pressure can be written as follows

$$
Q=\frac{\gamma}{2} \cdot p^{*} \cdot M^{2}
$$

where $p^{*}$ is the static pressure in the flow and $M$ is the free-stream Mach number. Now, after using (i) the right-hand side of Eq. (52) to replace the drag force in Eq. (48), and, (ii) the right-hand side of Eq. (53) to replace the dynamic pressure in Eq. (48), the drag coefficient in cruise condition becomes:

$$
c_{D} \approx \frac{2 \cdot W}{18 \cdot \gamma \cdot p^{*} \cdot A} \cdot M^{-2}
$$

The partial derivative of the drag coefficient with respect to the Mach number can easily be obtained from Eq. (54). We get the following result:

$$
\frac{\partial c_{D}}{\partial M} \approx \frac{2 \cdot W}{18 \cdot \gamma \cdot p^{*} \cdot A} \cdot(-2) \cdot M^{-3}
$$

It is convenient to use the absolute value of the partial derivative of the drag coefficient for the investigation of the relationship between the Mach number measurement error and the drag coefficient prediction error. Then, after computing the absolute value of both sides of Eq. (55), we get the following result:

$$
\left|\frac{\partial c_{D}}{\partial M}\right| \approx \frac{4 \cdot W}{18 \cdot \gamma \cdot p^{*} \cdot A} \cdot M^{-3}
$$

American Institute of Aeronautics and Astronautics 
Now, the relationship between the Mach number measurement error and the drag coefficient prediction error can be established. We get the following approximation:

\section{RELATIONSHIP BETWEEN DRAG COEFFICIENT AND MACH NUMBER ERROR}

$$
\Delta c_{D} \approx\left|\frac{\partial c_{D}}{\partial M}\right| \cdot \Delta M
$$

An estimate of the drag coefficient prediction error as a function of an assumed Mach number measurement error can be obtained if a realistic estimate of $\left|\partial c_{D} / \partial M\right|$ for a subsonic commercial transport aircraft in cruise condition is made. Again, similar to the approach taken in App. 1, it is assumed that (i) the ratio of the specific heats of air is $\gamma=1.4$, (ii) the cruise altitude is $h=10,000[\mathrm{~m}]$ and, (iii) the cruise Mach number equals $M=0.85$. In addition, we know from Ref. [1] that the barometric pressure at 10,000 [m] equals $p^{*}=26,496[P a]$. The total weight $(W)$ and reference area $(A)$ of typical subsonic commercial transport aircraft are given in Table 7 below. Therefore, all inputs on the right-hand side of Eq. (56) are known and the values of $\left|\partial c_{D} / \partial M\right|$ can be computed. Results of this calculation are listed in the last column of Table 7 .

Table 7: Partial derivative of cruise drag coefficient for existing aircraft $\left(g=9.81\left[\mathrm{~m} / \mathrm{sec}^{2}\right]\right)$.

\begin{tabular}{|c|c|c|c|c|}
\hline Aircraft Make/Model & $W / g$ & $W$ & $A$ & Eq. (56) $\Longrightarrow\left|\frac{\partial c_{D}}{\partial M}\right|$ \\
\hline \hline Boeing B747-400F & $396,895[\mathrm{~kg}]$ & $3,893,540[\mathrm{~N}]$ & $541\left[\mathrm{~m}^{2}\right]$ & 0.070 \\
\hline Boeing MD11F & $273,314[\mathrm{~kg}]$ & $2,681,210[\mathrm{~N}]$ & $339\left[\mathrm{~m}^{2}\right]$ & 0.077 \\
\hline Airbus A380F & $590,000[\mathrm{~kg}]$ & $5,787,900[\mathrm{~N}]$ & $845\left[\mathrm{~m}^{2}\right]$ & 0.067 \\
\hline
\end{tabular}

${ }^{\dagger}$ weight \& area taken from Ref. [9] ; ${ }^{\ddagger}$ weight \& area taken from Ref. [10]

It is sufficient for the current investigation to use the arithmetric mean of the predicted values of $\left|\partial c_{D} / \partial M\right|$ of the three aircraft listed in Table 7 because the value is only used to estimate the overall magnitude of the influence of an assumed Mach number measurement error on the drag coefficient prediction error. We get the following arithmetic mean of the partial derivative of the drag coefficient:

$$
\left|\frac{\partial c_{D}}{\partial M}\right| \approx 0.071
$$

Finally, a Mach number measurement error needs to be selected. The following choice is made:

$$
\Delta M= \pm 0.001
$$

Then, after using the values on the right-hand sides of Eqs. (58) and (59) on the right-hand side of Eq. (57), we get the following result:

$$
\Delta c_{D} \approx 0.071 \cdot \pm 0.001= \pm 0.000071 \approx \pm 0.0001 \equiv \pm \text { one drag count }
$$

The numerical result given in Eq. (60) can be summarized by the following statement:

A Mach number measurement error of $\approx 0.001$ results in a drag coefficient prediction error of $\approx 1$ count for a subsonic commercial transport aircraft that operates in cruise condition.

American Institute of Aeronautics and Astronautics 


\section{Appendix 3: Drag Prediction Precision and Angle of Attack Measurement}

In Appendix 1 is was rigorously shown that an objectively observed drag coefficient change of one count, i.e., $\Delta c_{D}=0.0001$, during the wind tunnel test of a subsonic commercial transport aircraft in cruise condition can have a noticeable impact on the prediction of the aircraft's payload. Therefore, the influence of different wind tunnel measurements on the drag coefficient calculation has to be well understood so that the total drag coefficient prediction error can be kept at or below one count.

The precision of the angle of attack measurement during a wind tunnel test is one input that influences the total drag coefficient prediction error. Therefore, it is useful to develop a relationship that directly relates an assumed small angle measurement change to a resulting small drag coefficient change because this relationship can be used to relate a known angle measurement error to a resulting drag coefficient prediction error. The derivation of such a relationship starts with the definition of the drag coefficient. It is defined as follows

$$
c_{D}=D /(Q \cdot A)
$$

where $D$ is the aerodynamic drag force, $Q$ is the dynamic pressure, and $A$ is the aircraft's reference area. The lift-to-drag ratio $L / D$ of a subsonic commercial transport aircraft in cruise condition is between 17 and 19 (see Ref. [11]). Therefore, the following approximate value of the lift-to-drag ratio can be introduced:

$$
L / D \approx 18
$$

Now, after solving the above equation for the drag force, we get the following relationship:

$$
D \approx L / 18
$$

In the next step, after using the right-hand side of Eq. (63) to replace the drag force in Eq. (61), we get the following approximation of the drag coefficient in cruise condition:

$$
c_{D} \approx \frac{1}{18} \cdot \frac{L}{Q \cdot A}
$$

The last fraction on the right-hand side of Eq. (64) equals the lift coefficient in cruise condition. Therefore, it is possible to simplify Eq. (64). We get the following relationship:

$$
c_{D} \approx \frac{1}{18} \cdot c_{L}
$$

It is possible to approximate the lift coefficient by using the lift slope and the angle of attack. The corresponding approximation has the following form:

$$
c_{L} \approx \frac{d c_{L}}{d \alpha} \cdot \alpha
$$

Then, after using the right-hand side of Eq. (66) to replace the lift coefficient in Eq. (65), we get the following equation:

$$
c_{D} \approx \frac{1}{18} \cdot \frac{d c_{L}}{d \alpha} \cdot \alpha
$$

The lift slope in cruise condition can be viewed as a constant as the angle of attack is somewhere between $3^{\circ}$ and $5^{\circ}$. Therefore, we get the following approximation:

$$
\text { cruise condition } \Longrightarrow \frac{d c_{L}}{d \alpha} \approx \text { const. }
$$

Consequently, the derivative of the drag coefficient in cruise condition with respect to the angle of attack can be computed to be:

$$
\frac{d c_{D}}{d \alpha} \approx \frac{1}{18} \cdot \frac{d c_{L}}{d \alpha}
$$


Finally, the relationship between angle measurement error and drag coefficient error can be defined by using the derivative of the drag coefficient with respect to the angle of attack. We get the following result:

\section{ANGLE MEASUREMENT ERROR AND DRAG COEFFICIENT ERROR}

$$
\Delta c_{D} \approx \frac{d c_{D}}{d \alpha} \cdot \Delta \alpha \approx \frac{1}{18} \cdot \frac{d c_{L}}{d \alpha} \cdot \Delta \alpha
$$

A realistic estimate of the lift slope for a typical subsonic commercial transport aircraft is needed so that the impact of an angle measurement error on the drag coefficient can be quantified. Known values of the aspect ratio $(\Lambda)$ and sweepback angle $(\psi)$ of the wing of a commercial transport aircraft may be combined with an assumed cruise Mach number and the subsonic similarity rules in order to get an estimate of the lift slope. Table 8 below explains different steps that are required for the calculation of the lift slope.

Table 8: Calculation of the lift slope $d c_{L} / d \alpha$ using the subsonic similarity rules.

\begin{tabular}{|c|c|c|c|}
\hline Step & Transformation Equation (inc. $\equiv$ incompressible value) & from Ref. [1] . & Eq. \\
\hline \hline 1 & $\Lambda_{\text {inc. }}=\Lambda \cdot \sqrt{1-M^{2}}$ & $\ldots$ Eq. (4-68b) & $(71 a)$ \\
\hline 2 & $\frac{1}{\tan \psi_{\text {inc. }}}=\frac{1}{\tan \psi} \cdot \sqrt{1-M^{2}}$ & $\ldots$ Eq. (4-68c) & $(71 b)$ \\
\hline 3 & $\left(\frac{d c_{L}}{d \alpha}\right)_{\psi=0^{\circ}}=\frac{\pi \Lambda_{\text {inc. }}}{\sqrt{\Lambda_{\text {inc. }}^{2} / 4+1}+1}$ & $\ldots$ Eq. (3-98) & $(71 c)$ \\
\hline 4 & $\left(\frac{d c_{L}}{d \alpha}\right)_{\psi=\psi_{i n c .}}=\left(\frac{d c_{L}}{d \alpha}\right)_{\psi=0^{\circ}} \cos \psi_{\text {inc. }}$ & $\ldots$ Eq. (3-123) & $(71 d)$ \\
\hline 5 & $\frac{d c_{L}}{d \alpha}=\frac{1}{\sqrt{1-M^{2}} \cdot\left(\frac{d c_{L}}{d \alpha}\right)_{\psi=\psi_{i n c .}}}$ & $\ldots$ p. 264, Table 4-4 & $(71 e)$ \\
\hline
\end{tabular}

Now, it is possible to apply the similarity rules to three different subsonic commercial transport aircraft. Table 9 below lists the inputs needed for the calculation of the lift slope assuming that the chosen cruise Mach number is 0.85 (these values were obtained from Refs. [9] and [10]).

Table 9: Aspect ratio and sweepback angle of typical subsonic commercial transport aircraft.

\begin{tabular}{|c|c|c|c|}
\hline & Boeing B747-400F & Boeing MD11F & Airbus A380 \\
\hline \hline Aspect Ratio $(\Lambda)$ & 7.7 & 7.9 & 7.5 \\
\hline Sweepback Angle $(\psi)$ & $37.5^{\circ}$ & $35.0^{\circ}$ & $33.5^{\circ}$ \\
\hline
\end{tabular}

American Institute of Aeronautics and Astronautics 
Table 10 below lists the result of the calculation of the lift slope for the three chosen aircraft.

Table 10: Estimates of the lift slope $d c_{L} / d \alpha$ for different aircraft.

\begin{tabular}{|c|c|c|c|}
\hline Computed Value for $M=0.85$ & Boeing B747-400F & Boeing MD11F & Airbus A380 \\
\hline \hline Eq. $(71 a) \Longrightarrow \quad \Lambda_{\text {inc. }}$ & 4.06 & 4.16 & 3.95 \\
\hline Eq. $(71 b) \Longrightarrow\left(\psi_{\text {inc. }}\right.$ & $55.53^{\circ}$ & $53.04^{\circ}$ & $51.48^{\circ}$ \\
\hline Eq. $(71 c) \Longrightarrow\left(\frac{d c_{L}}{d \alpha}\right)_{\psi=0^{\circ}}$ & 3.91 & 3.95 & 3.86 \\
\hline Eq. $(71 d) \Longrightarrow\left(\frac{d c_{L}}{d \alpha}\right)_{\psi=\psi_{\text {inc. }}}$ & 2.21 & 2.38 & 2.41 \\
\hline Eq. $(71 e) \Longrightarrow\left(\frac{d c_{L}}{d \alpha}\right.$ & 4.20 & 4.51 & 4.57 \\
\hline
\end{tabular}

The overall magnitude of the authors' theoretical lift slope estimates for the three chosen aircraft can be validated by comparing them with results for similar aircraft that are reported in the literature. For example, lift slopes of 4.41 and 4.90 are reported in NASA CR-2144 for a Convair CV-880M aircraft that traveled at a Mach number of 0.86 at 23,000 and 35,000 ft (see Ref. [12], p. 198). These values are reasonably close to the values that are reported in the last row of Table 10 above considering the fact that the authors' theoretical lift slope estimates do not include the lift contribution from the horizontal tail, fuselage, and jet engines. The authors decided to simply use the arithmetic mean of the three lift slopes reported in Table 10 as input for the evaluation of the right-hand side of Eq. (70). Then, we get the following average value:

$$
\frac{d c_{L}}{d \alpha} \approx 4.43
$$

In addition, it is assumed that the angle measurement error equals \pm 0.02 degrees. Then, we get:

$$
\Delta \alpha= \pm 0.000349 \text { radians } \equiv \pm 0.02^{\circ}
$$

Now, the right-hand side of Eq. (70) can be evaluated. We get the following estimate of the drag coefficient prediction error as a function of the assumed angle measurement error of \pm 0.02 degrees:

$$
\Delta c_{D} \approx \frac{1}{18} \cdot 4.43 \cdot \pm 0.000349= \pm 0.000086 \approx \pm 0.0001 \equiv \pm \text { one drag count }
$$

Finally, the numerical result given in Eq. (74) can be summarized as follows:

An angle measurement error of $\approx 0.02$ degrees results in a drag coefficient prediction error of $\approx 1$ count for a subsonic commercial transport aircraft that operates in cruise condition.

American Institute of Aeronautics and Astronautics 


\section{Appendix 4: Precision Error Estimate for Aerodynamic Coefficients}

One of the primary goals of a wind tunnel test is the experimental determination of aerodynamic coefficients. These dimensionless quantities describe magnitude and sign of aerodynamic loads that act on a test article. A pre-test estimate of the precision error of an aerodynamic coefficient can easily be obtained after generalizing the approach that is described in this paper for the drag coefficient.

In principle, an aerodynamic coefficient is a dimensionless description of the magnitude and sign of a force or moment that acts on the wind tunnel model. The acting force or moment is made dimensionless after dividing it by the dynamic pressure and a reference parameter. This definition can be written as follows

\section{DEFINITION OF AN AERODYNAMIC COEFFICIENT}

$$
\begin{gathered}
c_{\xi}(\xi, Q)=\frac{\xi}{Q \cdot A_{\xi}} \\
\text { where } \\
A_{\xi} \equiv\left\{\begin{array}{cll}
A & \Longleftrightarrow & \text { if } c_{\xi} \text { is a force coefficient } \\
A \cdot l & \Longleftrightarrow \text { if } c_{\xi} \text { is a moment coefficient }
\end{array}\right.
\end{gathered}
$$

where $\xi$ represents an aerodynamic force or moment, $Q$ is the dynamic pressure, $A$ is a reference area, and $l$ is a reference length. The coefficient defined in Eq. (75a) above is a multivariate function of two independent variables, i.e., $\xi$ and $Q$, because the reference parameter $A_{\xi}$ is a constant that is only related to the geometry of the test article. Consequently, the total differential of the coefficient can be described as follows

$$
d c_{\xi}=\frac{\partial c_{\xi}}{\partial \xi} \cdot d \xi+\frac{\partial c_{\xi}}{\partial Q} \cdot d Q=\underbrace{\frac{1}{Q \cdot A_{\xi}} \cdot d \xi}_{Q=\text { const. }}+\underbrace{\left(-c_{\xi}\right) \cdot \frac{d Q}{Q}}_{\xi=\text { const. }}
$$

where $d c_{\xi}, d \xi$, and $d Q$ represent small changes of the related variables. Now, assuming that small random changes of the tunnel conditions, the model support system orientation, and the electrical outputs of the balance gages are primarily responsible for the fact that the observed aerodynamic coefficients of two repeat points differ by a small amount, it is possible to directly relate them to small changes of the independent variables of the coefficient. Then, an upper bound $\delta c_{\xi}$ of the coefficient's precision error can be defined by using the absolute values of the partial derivatives of the coefficient in combination with (i) the absolute value $|\delta \xi|$ of small random changes of the measured force or moment and (ii) the absolute value $|\delta Q|$ of small random changes of the dynamic pressure. Consequently, we get the following "conservative" estimate of the upper bound of the coefficient's precision error:

$$
\delta c_{\xi} \leq \underbrace{\frac{1}{Q \cdot A_{\xi}} \cdot|\delta \xi|}_{Q=\text { const. }}+\underbrace{\left|c_{\xi}\right| \cdot\left|\frac{\delta Q}{Q}\right|}_{\xi=\text { const. }}
$$

The above estimate of the precision error of an aerodynamic coefficient has two parts that lend themselves to interpretation. The first part is a contribution that is related to the balance and support system characteristics as the precision error $|\delta \xi|$ of the force or moment measurement appears. The second part is related to the measurement of the tunnel conditions as the relative error $|\delta Q / Q|$ of the dynamic pressure measurement appears as multiplier. Therefore, Eq. (76b) can be summarized as follows: 


\section{UPPER BOUND OF PRECISION ERROR OF AN AERODYNAMIC COEFFICIENT}

$$
\begin{aligned}
\delta c_{\xi}(\delta \xi, \delta Q) & \leq\left.\delta c_{\xi}\right|_{Q=\text { const. }}+\left.\delta c_{\xi}\right|_{\xi=\text { const. }} \\
& \text { where } \\
\text { balance and/or support system choice } & \left.\Longrightarrow \delta c_{\xi}\right|_{Q=\text { const. }} \leq \frac{1}{Q \cdot A_{\xi}} \cdot|\delta \xi| \\
\text { tunnel condition choice } & \left.\Longrightarrow \delta c_{\xi}\right|_{\xi=\text { const. }} \leq\left|c_{\xi}\right| \cdot\left|\frac{\delta Q}{Q}\right|
\end{aligned}
$$

The above interpretation highlights the fact that the precision error $\delta c_{\xi}$ of an aerodynamic coefficient, similar to the coefficient itself, can be expressed as a function of two independent variables. They are the precision error $|\delta \xi|$ of the measurement of the aerodynamic load and the precision error $|\delta Q|$ of the dynamic pressure measurement.

The input variables needed for the calculation of the precision error of an aerodynamic coefficient can be split into two groups. The first group consists of two variables that are related to the dynamic pressure that the test article experiences. They are the dynamic pressure $Q$ and the estimate $|\delta Q|$ of the precision error of the dynamic pressure measurement. The second group consist of three variables that are related to the force or moment measurement and the geometry of the test article. They are the aerodynamic coefficient $c_{\xi}$, the test article's reference parameter $A_{\xi}$, and the estimate $|\delta \xi|$ of precision error of the force or moment measurement. It is possible to directly identify the three variables of the second group for different straingage balance load formats that are used in the aerospace testing community. For example, the following

\begin{tabular}{|c|c|c|c|}
\hline$c_{\xi}$ & $\xi$ & $A_{\xi}$ & $|\delta \xi| \equiv$ Precision Error of Aerodynamic Force or Moment \\
\hline$c_{L}$ & $L$ & $A$ & $|\delta \xi|=|\delta A F|^{\dagger} \cdot \sin |\alpha|+|\delta N F|^{\dagger} \cdot \cos |\alpha|$ \\
\hline$c_{D}$ & $D$ & $A$ & $|\delta \xi|=|\delta A F|^{\dagger} \cdot \cos |\alpha|+|\delta N F|^{\dagger} \cdot \sin |\alpha|$ \\
\hline$c_{M}$ & $P$ & $A \cdot d$ & $|\delta \xi|=|\delta P M|^{\dagger}$ \\
\hline
\end{tabular}
inputs are identified for the lift, drag, and pitching moment coefficient if it is assumed that the balance describes loads in direct-read format:

Table 11: Inputs for the precision error calculation for a direct-read balance.

It is important to point out that the precision error estimate $|\delta \alpha|$ of the angle measurement associated with the lift and drag coefficient is implicitly contained in the precision error estimates $|\delta A F|$ and $|\delta N F|$ of the related balance load measurements (see explanations associated with the introduction of Eqs. (13b) to $(16 b)$ for more detail). In addition, it is assumed that the precision errors $|\delta A F|,|\delta N F|$, and $|\delta P M|$ of the balance load measurements can be described as the product of an observed random error $|\varphi| \approx 1[\mu V / V]$ of the bridge output measurements with an estimate $S\left(F_{i}, R_{1}, \ldots, R_{n}\right)$ of the upper bound of the partial derivatives of the force or moment with respect to all bridge outputs (see Eq. (13b) and (13c)).

Table 12 below shows the inputs for the lift, drag, and pitching moment coefficient of a balance that

American Institute of Aeronautics and Astronautics 
describes loads in force balance format. The required total normal force is obtained after transforming the forward and aft normal force components from force balance to direct-read format (see, e.g., Ref. [5] for a derivation of the load transformations for a force balance). It is important to point out that the "+" operator and absolute values of all load components must be used for the definition of the precision errors because the sign of the different precision error contributions is unknown.

Table 12: Inputs for the precision error calculation for a force balance. ${ }^{\dagger}$

\begin{tabular}{|c|c|c|c|}
\hline$c_{\xi}$ & $\xi$ & $A_{\xi}$ & $|\delta \xi| \equiv$ Precision Error of Aerodynamic Force or Moment \\
\hline \hline$c_{L}$ & $L$ & $A$ & $|\delta \xi|=|\delta A F|^{\ddagger} \cdot \sin |\alpha|+\left\{|\delta N 1|^{\ddagger}+|\delta N 2|^{\ddagger}\right\} \cdot \cos |\alpha|$ \\
\hline$c_{D}$ & $D$ & $A$ & $|\delta \xi|=|\delta A F|^{\ddagger} \cdot \cos |\alpha|+\left\{|\delta N 1|^{\ddagger}+|\delta N 2|^{\ddagger}\right\} \cdot \sin |\alpha|$ \\
\hline$c_{M}$ & $P$ & $A \cdot d$ & $|\delta \xi|=\left\{|\delta N 1|^{\ddagger}+|\delta N 2|^{\ddagger}\right\} \cdot(b / 2)$ \\
\hline
\end{tabular}

${ }^{\dagger}$ It is assumed that the balance moment center is halfway between the forward and aft gages. ${ }^{\ddagger}|\delta A F|,|\delta N 1|,|\delta N 2| \equiv\left|\delta F_{i}\right| \Longleftrightarrow\left|\delta F_{i}\right|=|\varphi| \cdot S\left(F_{i}, R_{1}, \ldots, R_{n}\right)$; see Eq. (13b), (13c).

Table 13 below shows the inputs for the lift, drag, and pitching moment coefficient of a balance that describes loads in moment balance format. The required total normal force is obtained after transforming the forward and aft pitching moment components from moment balance to direct-read format (see, e.g., Ref. [5] for a derivation of the load transformations for a moment balance). Again, as it was the case for a force balance, the "+" operator and absolute values of all load components must be used for the definition of the precision errors because the sign of the different precision error contributions is unknown.

Table 13: Inputs for the precision error calculation for a moment balance. ${ }^{\dagger}$

\begin{tabular}{|c|c|c|c|}
\hline$c_{\xi}$ & $\xi$ & $A_{\xi}$ & $|\delta \xi| \equiv$ Precision Error of Aerodynamic Force or Moment \\
\hline \hline$c_{L}$ & $L$ & $A$ & $|\delta \xi|=|\delta A F|^{\ddagger} \cdot \sin |\alpha|+\frac{|\delta P M 1|^{\ddagger}+|\delta P M 2|^{\ddagger}}{b} \cdot \cos |\alpha|$ \\
\hline$c_{D}$ & $D$ & $A$ & $|\delta \xi|=|\delta A F|^{\ddagger} \cdot \cos |\alpha|+\frac{|\delta P M 1|^{\ddagger}+|\delta P M 2|^{\ddagger}}{b} \cdot \sin |\alpha|$ \\
\hline$c_{M}$ & $P$ & $A \cdot d$ & $|\delta \xi|=\frac{|\delta P M 1|^{\ddagger}+|\delta P M 2|^{\ddagger}}{2}$ \\
\hline
\end{tabular}

${ }^{\dagger}$ It is assumed that the balance moment center is halfway between the forward and aft gages. $\ddagger|\delta A F|,|\delta P M 1|,|\delta P M 2| \equiv\left|\delta F_{i}\right| \Longleftrightarrow\left|\delta F_{i}\right|=|\varphi| \cdot S\left(F_{i}, R_{1}, \ldots, R_{n}\right)$; see Eq. (13b), (13c).

Precision error estimates for other aerodynamic coefficients (side force coefficient, rolling moment coefficient, yawing moment coefficient) can be obtained by following the general approach that is outlined above for the lift, drag, and pitching moment coefficients. 